\title{
Faster Parallel Algorithm for Approximate Shortest Path
}

\author{
Jason Li \\ jmli@cs.cmu.edu \\ Carnegie Mellon University \\ Pittsburgh, PA, USA
}

\begin{abstract}
We present the first $m$ polylog $(n)$ work, $\operatorname{polylog}(n)$ time algorithm in the PRAM model that computes $(1+\epsilon)$-approximate singlesource shortest paths on weighted, undirected graphs. This improves upon the breakthrough result of Cohen [JACM'00] that achieves $O\left(m^{1+\epsilon_{0}}\right)$ work and polylog $(n)$ time. While most previous approaches, including Cohen's, leveraged the power of hopsets, our algorithm builds upon the recent developments in continuous optimization, studying the shortest path problem from the lens of the closely-related minimum transshipment problem. To obtain our algorithm, we demonstrate a series of near-linear work, polylogarithmictime reductions between the problems of approximate shortest path, approximate transshipment, and $\ell_{1}$-embeddings, and establish a recursive algorithm that cycles through the three problems and reduces the graph size on each cycle. As a consequence, we also obtain faster parallel algorithms for approximate transshipment and $\ell_{1}$-embeddings with polylogarithmic distortion. The minimum transshipment algorithm in particular improves upon the previous best $m^{1+o(1)}$ work sequential algorithm of Sherman [SODA'17].

To improve readability, the paper is almost entirely self-contained, save for several staple theorems in algorithms and combinatorics.
\end{abstract}

\section{CCS CONCEPTS}

- Theory of computation $\rightarrow$ Parallel algorithms; Shortest paths; Streaming, sublinear and near linear time algorithms.

\section{KEYWORDS}

Parallel Algorithms, Shortest Path, Minimum Transshipment

\section{ACM Reference Format:}

Jason Li. 2020. Faster Parallel Algorithm for Approximate Shortest Path. In Proceedings of the 52nd Annual ACM SIGACT Symposium on Theory of Computing (STOC '20), June 22-26, 2020, Chicago, IL, USA. ACM, New York, NY, USA, 14 pages. https://doi.org/10.1145/3357713.3384268

\section{INTRODUCTION}

The single-source shortest path problem is one of the most fundamental combinatorial optimization problems, and is also among the most notorious in parallel computation models. While the sequential model has simple near-linear time algorithm dating back to

Permission to make digital or hard copies of all or part of this work for personal or classroom use is granted without fee provided that copies are not made or distributed for profit or commercial advantage and that copies bear this notice and the full citation on the first page. Copyrights for components of this work owned by others than ACM must be honored. Abstracting with credit is permitted. To copy otherwise, or republish, to post on servers or to redistribute to lists, requires prior specific permission and/or a fee. Request permissions from permissions@acm.org.

STOC '20, June 22-26, 2020, Chicago, IL, USA

(C) 2020 Association for Computing Machinery.

ACM ISBN 978-1-4503-6979-4/20/06 . \$ \$15.00

https://doi.org/10.1145/3357713.3384268
Dijkstra, much remains unknown for even the PRAM model despite decades of extensive research.

One of the most well-known settings studied so far in the PRAM model is the case of $(1+\epsilon)$-approximate single-source shortest paths in undirected graphs. Early work on this problem produced algorithms in sublinear time [KS92, KS97], until the breakthrough result of Cohen [Coh00], who presented an algorithm in $O\left(m^{1+\epsilon_{0}}\right)$ work (for any constant $\epsilon_{0}>0$ ) and $\operatorname{polylog}(n)$ time through the use of hopsets: additional edges added to the graph so that short paths in the graph span few edges. Since then, it was a long-standing open problem whether Cohen's algorithm could be improved to run in $m$ polylog $(n)$ work while keeping the time polylog $(n)$.

Recently, this question was partially answered by Abboud, Bodwin and Pettie, surprisingly in the negative: they showed that there exist families of graphs for which any hopsets on these graphs must have size $\Omega\left(m^{1+\epsilon_{0}}\right)$, thereby lower bounding the work by $\Omega\left(m^{1+\epsilon_{0}}\right)$ for any purely hopset-based algorithm like Cohen's. While their lower bound does not rule out other approaches to this problem, no other directions of attack have come close to matching Cohen's method of hopsets.

In this paper, we tackle this problem from a new perspective: continuous optimization, especially the methods pioneered by Sherman [She13] for the maximum flow problem. By reducing to studying the closely-related and more continuous minimum transshipment problem, we provide the first $(1+\epsilon)$-approximate SSSP algorithm for weighted, undirected graphs in $m$ polylog $(n)$ work and polylog $(n)$ time in the PRAM model, bypassing the hopset lower bound and resolving the aforementioned open problem. This serves as evidence that continuous optimization, with its rich theory in graph algorithm and inherent parallelism, is a promising research direction in parallel graph algorithms and can bypass known barriers to other common approaches.

Theorem 1.1 (PARAllel SSSP). There exists a parallel algorithm that, given an undirected graph with nonnegative weights, computes a $(1+\epsilon)$-approximate single-source shortest path tree in $m \operatorname{polylog}(n) \epsilon^{-2}$ work and $\operatorname{polylog}(n) \epsilon^{-2}$ time in the PRAM model.

Our SSSP algorithm is recursive, cycling through three problems in a round-robin fashion: SSSP, transshipment, and the problem of computing an $\ell_{1}$-embedding of a graph with polylog $(n)$ distortion in $O(\log n)$ dimensions. That is, each problem calls the next problem on the cyclic list possibly many times, and possibly on a smaller graph instance. Hence, we obtain parallel algorithms with similar running times for the other two problems as well.

Theorem 1.2 (PARAllel transshipment). There exists a parallel algorithm that, given an undirected graph with nonnegative weights and polynomial aspect ratio, computes $a(1+\epsilon)$-approximation to minimum transshipment in $m \operatorname{polylog}(n) \epsilon^{-2}$ work and $\operatorname{polylog}(n) \epsilon^{-2}$ time in the PRAM model. 
THEOREM 1.3 (PARALLEL $\ell_{1}$-EMBEDDING). There exists a parallel algorithm that, given an undirected graph with nonnegative weights and polynomial aspect ratio, computes an $\ell_{1}$-embedding with $\operatorname{polylog}(n)$ distortion in $O(\log n)$ dimensions in $m$ polylog $(n)$ work and $\operatorname{polylog}(n)$ time in the PRAM model.

Theorem 1.2 also establishes the first $m$ polylog $(n)$ time sequential algorithm for $(1+\epsilon)$-approximate transshipment, improving upon the $m^{1+o(1)}$-time algorithm of Sherman [She17b]. For readers primarily interested in the sequential setting, we further optimize our parameters to the following. Note that the best algorithm for the closely-related maximum flow problem [Pen16] requires $O\left(m \log ^{41} n\right)$ time in comparison. Our algorithm is also technically considerably simpler than the maximum flow algorithm, and may serve as a gentler introduction to readers new to continuous optimization methods in graph algorithms.

Theorem 1.4 (Sequential transshipment). There is an algorithm that, given an undirected graph with nonnegative weights and polynomial aspect ratio, computes $a(1+\epsilon)$-approximation to minimum transshipment in time $O\left(\left(m \log ^{10} n+n \log ^{15} n\right) \epsilon^{-2}(\log \log n)^{O(1)}\right)$.

\subsection{Our Techniques}

Our recursive algorithm is inspired by a similar recursive algorithm by Peng [Pen16] for maximum flow. It is instructive to compare our result to that of Peng [Pen16], the first $\widetilde{O}(m)$ time $^{1}$ algorithm for $(1-\epsilon)$-approximate maximum flow. ${ }^{2}$ Peng [Pen16] uses an oblivious routing scheme for maximum flow that achieves polylog $(n)$-approximation, but requires polylog $(n)$ calls to $(1-\epsilon)$ maximum flow [RST14]. This oblivious routing scheme produced a chicken-and-egg situation for maximum flow and oblivious routing, since each one required calls to the other. Peng's main contribution is breaking this cycle, by allowing the oblivious routing to call maximum flow on sufficiently smaller-sized graphs to produce an efficient recursive algorithm. Here, we adopt a similar recursive approach, cycling through the problems of shortest path, minimum transshipment, oblivious routing, and $\ell_{1}$-embedding.

Step 1: reduce to transshipment. The first step of the algorithm is to reduce the approximate SSSP problem to the approximate minimum transshipment problem, which was previously done in [BKKL16] for various other computational models. Making it work in the PRAM model requires a little more care, and for completeness, we provide a self-contained reduction in the full version of this paper.

Note that if we were in the exact case, then the reduction would be immediate: there is a straightforward reduction from exact SSSP to exact transshipment: set $-(n-1)$ demand on the source vertex and +1 demand on the rest, and from the transshipment flow we can recover the exact SSSP relatively easily. However, in the approximate case, an approximate transshipment solution in the same

\footnotetext{
${ }^{1}$ Throughout the paper, we use the standard $\widetilde{O}(\cdot)$ notation to hide polylogarithmic factors in the running time.

${ }^{2}$ Note that maximum flow and minimum transshipment are closely related: for graph incidence matrix $A$ and a diagonal matrix $C$ capturing the edge capacities/costs, and for a given demand vector $b$, the maximum flow problem is equivalent to $\min \|f\|_{\infty}$ subject to $A f=b$, and the minimum transshipment problem is exactly min $\|f\|_{1}$ subject to $A f=b$.
}

reduction only satisfies distances on "average". [BKKL16] handles this issue through $O(\log n)$ calls to approximate transshipment with carefully and adaptively constructed demands on each call; we use $O\left(\log ^{2} n\right)$ calls instead with a more sophisticated reduction.

Step 2: $\ell_{1}$-oblivious routing. Sherman's framework reduces the problem of approximate transshipment to that of $\ell_{1}$-oblivious routing, which we define later. For readers familiar with traditional oblivious routing (for maximum flow) which we will henceforth call $\ell_{\infty}$-oblivious routing, the $\ell_{1}$-oblivious routing problem is the same except the cost is measured by the total (weighted) sum of congestions on edges rather than the maximum congestion. and our main technical contribution of the paper.

Just like $\ell_{\infty}$-oblivious routing is harder than maximum flow, $\ell_{1}$-oblivious routing is harder than transshipment. However, the benefit to considering $\ell_{1}$ - or $\ell_{\infty}$-oblivious routing is that only a polylog $(n)$-approximate solution is needed to obtain a $(1+\epsilon)$ approximate maximum flow or transshipment solution; that is, Sherman's framework can be thought of as boosting the error from polylog $(n)$ to $(1+\epsilon)$ (at the expense of solving a harder problem).

In [She17b], Sherman uses his framework to solve $(1+\epsilon)$ approximate transshipment in $m^{1+o(1)}$ sequential time by providing an $m^{o(1)}$-approximate $\ell_{1}$-oblivious routing scheme that runs in $m^{1+o(1)}$ time. Our main technical contribution in this entire paper is providing an improved scheme that is polylog( $n)$-approximate and runs in $m$ polylog $(n)$ time. Like Sherman, our algorithm requires an initial $\ell_{1}$-embedding of the vertices of the graph to establish some geometric structure on the vertices.

The $\ell_{1}$-oblivious routing algorithm is mostly self-contained and has no relation to the parallel sections of the paper. We therefore isolate it in its own section, Section 4, for the convenience of readers primarily interested in transshipment in the sequential setting.

Step 3: $\ell_{1}$-embedding and ultra-sparsification. If we could compute an $\ell_{1}$-embedding with polylog $(n)$ distortion, then we would be done. (We can obtain $O(\log n)$ dimensions for free with JohnsonLindenstrauss dimension reduction.) Unfortunately, while this task is simple to compute sequentially, no work-efficient parallel algorithm is known. This is because the popular algorithms that compute $\ell_{1}$-embeddings sequentially all require distance computations as subroutines, and no work-efficient parallel algorithm for SSSP is known. (If one were known, then there would be no need for our result in the first place!)

Recall that we sought out to solve SSSP, and currently, our $\ell_{1}$ embedding problem requires an SSSP routine on its own. This is where Peng's key insight comes to play: while recursing naively on the same graph will not work (since it would loop endlessly), if we can recurse on sufficiently smaller graphs, then the recursion analysis would produce an algorithm with the desired running time. This is indeed Peng's approach for maximum flow: he makes one maximum flow instance call $\ell_{\infty}$-oblivious routing, which in turn calls maximum flow a number of times, but ensures that the total size of the recursive calls is at most half the size of the original graph. The recursion then works out to roughly $T(m)=\sum_{i} T\left(m_{i}\right)+\widetilde{O}(m)$ where $\sum_{i} m_{i} \leq m / 2$, which solves to $T(m)=\widetilde{O}(m)$. 
How does Peng achieve the reduction in size? Instead of computing $\ell_{\infty}$-oblivious routing in the original graph $G$, he first (edge)sparsifies $G$ into a graph $H$ on $n$ vertices and $(n-1)+O\left(\frac{m}{\operatorname{polylog}(n)}\right)$ edges by computing an ultra-sparsifier of the graph [KMP14a]. This is a graph that is so sparse that it is almost "tree-like" (at least when $m=\widetilde{O}(n))$. Of course, this alone might not achieve the desired size reduction, for example if $m \approx n$. Therefore, he next vertex-sparsifies $H$ into a graph $H^{\prime}$ with $O\left(\frac{m}{\operatorname{polylog}(n)}\right)$ vertices and $O\left(\frac{m}{\text { polylog(n) }}\right)$ edges using a $j$-tree construction of Madry [Mad10]. He now calls $\ell_{\infty}$-oblivious routing on $H^{\prime}$ (instead of $G$ ), which again calls maximum flow, but this time on graphs of small enough size (w.r.t. the original graph) to make the recursion work out. Moreover, by the properties of the ultra-sparsifier and the vertex-sparsifier, a polylog $(n)$-approximate $\ell_{\infty}$-oblivious routing scheme for $H^{\prime}$ is also a polylog $(n)$-approximate $\ell_{\infty}$-oblivious routing scheme for $G$ (that is, the approximation suffers an extra polylog $(n)$ factor). The specific $\operatorname{polylog}(n)$ factor does not matter at the end, since in Sherman's framework, any polylog $(n)$ factor is sufficient to boost the error to $(1+\epsilon)$ for maximum flow at an additional additive cost of $\widetilde{O}(m)$.

Our approach is similar, but adapted from $\ell_{\infty}$ /maximum flow to $\ell_{1}$ /transshipment. The $\ell_{1}$-analogy of an ultrasparsifier has been studied previously by Elkin and Neiman [EN18], who coined the term ultra-sparse spanner; in this paper, we will use ultra-spanner instead to emphasize its connection to ultra-sparsifiers. Instead of running $\ell_{1}$-embedding on $G$, we compute an ultra-spanner $H$, and then vertex-sparsify it in the same manner as Peng; again, the resulting graph $H^{\prime}$ has $O\left(\frac{m}{\operatorname{polylog}(n)}\right)$ vertices and edges. We then run $\ell_{1}$-embedding on $H^{\prime}$, making calls to (approximate) SSSP on graphs of much smaller size. It turns out that approximate SSSP works for the $\ell_{1}$-embedding algorithm that we use, provided that the distances satisfy a certain triangle inequality condition that our SSSP algorithm obtains for free.

\subsection{Concurrent Work: [ASZ19]}

At around the same time, Andoni, Stein, and Zhong [ASZ19] obtained similar results, solving approximate shortest paths in the PRAM model in $\widetilde{O}(m)$ work and polylog $(n)$ time. Their algorithm only computes an $(1+\epsilon)$-approximate $s-t$ path, while ours works for full SSSP. Both algorithms are based on transshipment and Sherman's framework, but they deviate in the general algorithmic structure. Our routing algorithm recursively calls itself and reduces the sizes of the recursive calls via ultra-spanners, while they develop the concepts of subemulators and compressed preconditioners to construct the oblivious routing (or preconditioner) directly.

While both results were arXived on the same day, our result had a nontrivial omission that was patched a month and a half later. The author acknowledges that the work of Andoni et al. was completed first, and ours is a chronologically later but slightly stronger result. See the Acknowledgments for more details.

\section{PRELIMINARIES}

All graphs in the paper are undirected and (positively) weighted. Given a graph $G$, we define $V(G)$ and $E(G)$ as the vertices and edges of the graph. For two vertices $u, v \in V(G)$, we define $d_{G}(u, v)$ as the (weighted) distance between $u$ and $v$ in $G$; if the graph $G$ is clear from context, we sometimes use $d(u, v)$ instead.

\subsection{Transshipment Preliminaries}

The definitions below are central for our sequential transshipment algorithm (Theorem 1.4, Section 4) and are also relevant for the parallel algorithms.

Definition 2.1 (Transshipment). The minimum transshipment problem inputs a (positively) weighted, undirected graph $G=(V, E)$, and defines the following auxiliary matrices:

(1) Adjacency matrix $A \in \mathbb{R}^{V \times E}$ : for each edge $e=(u, v)$, the column of $A$ indexed by e equals either $1_{u}-1_{v}$ or $1_{v}-1_{u}$.

(2) Cost matrix $C \in \mathbb{R}^{E \times E}:$ a diagonal matrix with entry $C_{e, e}$ equal to the weight of edge e.

In a transshipment instance, we are also given a demand vector $b \in \mathbb{R}^{V}$ satisfying $\mathbf{1}^{T} b=0$.

Consider now the LP formulation for minimum transshipment: $\min \|C f\|_{1}: A f=b$, and its dual, $\max b^{T} \phi:\left\|C^{-1} A^{T} \phi\right\|_{\infty} \leq 1$. Let us define the solutions to the primal and dual formulations as flows and potentials:

Definition 2.2 (Flow). Given a transshipment instance, $a$ flow vector (or flow) is a vector $f \in \mathbb{R}^{E}$ satisfying the primal constraints $A f=b$, and it has cost $\|C f\|_{1}$. The flow minimizing $\|C f\|_{1}$ is called the optimal flow of the transshipment instance. For any $\alpha \geq 1$, an $\alpha$ approximate flow is a flow whose value $\|C f\|_{1}$ is at most $\alpha$ times the minimum possible (over all flows).

Definition 2.3 (Potential). Given a transshipment instance, a set of potentials (or potential) is a vector $\phi \in \mathbb{R}^{V}$ satisfying the dual constraints $\left\|C^{-1} A^{T} \phi\right\|_{\infty} \leq 1$. The potential maximizing $b^{T} \phi$ is called the optimal potential of the transshipment instance.

For convenience, we will treat potentials as functions on $V$; that is, we will use the notation $\phi(v)$ instead of $\phi_{v}$.

Definition 2.4 (Flow-potential pair). For any flow $f \in \mathbb{R}^{E}$ and potential $\phi \in \mathbb{R}^{V}$, the pair $(f, \phi)$ is called a flow-potential pair. For $\alpha \geq 1,(f, \phi)$ is an $\alpha$-approximate flow-potential pair if $\|C f\|_{1} \leq$ $\alpha b^{T} \phi$.

Fact 2.5. If $(f, \phi)$ is an $\alpha$-approximate flow-potential pair, then $f$ is an $\alpha$-approximate flow.

Proof. Let $f^{*}$ be the optimal flow. The two LPs min $\|C f\|_{1}$ : Af $=b$ and $\max b^{T} \phi:\left\|C^{-1} A^{T} \phi\right\|_{\infty} \leq 1$ are duals of each other, so by (weak) LP duality, the potential $\phi$ satisfies $b^{T} \phi \leq\left\|C f^{*}\right\|_{1}$. Since $(f, \phi)$ is an $\alpha$-approximate flow-potential pair, we have $\|C f\|_{1} \leq$ $\alpha b^{T} \phi \leq \alpha\left\|C f^{*}\right\|_{1}$.

Definition 2.6 (opt). Given a transshipment problem and demand vector $b$, define opt $(b)$ as the cost of the optimal flow of that instance, that is:

$$
\operatorname{opt}(b):=\min _{f: A f=b}\|C f\|_{1} .
$$

When the underlying graph $G$ is ambiguous, we use the notation opt $_{G}(b)$ instead. 


\subsection{Parallel Shortest Path Preliminaries}

The definitions below are confined to the parallel algorithms in the paper, so a reader primarily interested in the sequential transshipment algorithm (Theorem 1.4, Section 4) may skip these.

We first introduce a notion of approximate SSSP distances which we call approximate SSSP potentials.

Definition 2.7 (Approximate $s$-SSSP potential). Given a graph $G=(V, E)$ and a source $s$, a vector $\phi \in \mathbb{R}^{V}$ is an $\alpha$-approximate $s$-SSSP potential if:

(1) For all $v \in V, \phi(v)-\phi(s) \geq \frac{1}{\alpha} \cdot d(s, v)$

(2) For each edge $(u, v),|\phi(u)-\phi(v)| \leq w(u, v)$.

When the source $s$ is either irrelevant or clear from context, we may use $\alpha$-approximate SSSP potential (without the s) instead.

Observe that the approximate SSSP potential problem is slightly more stringent than simply approximate shortest path distances: the second condition of Definition 2.7 requires that distances satisfy a sort of approximate subtractive triangle inequality. To illustrate why this condition is more restrictive, imagine a graph on three vertices $s, u, v$, with $d(s, u)=d(s, v)=100$ and $d(u, v)=1$, and let $\alpha:=10 / 9$. Then, the distance estimates $\tilde{d}(s)=0$ and $\tilde{d}(u)=90$ and $\tilde{d}(v)=100$ are $\alpha$-approximate SSSP distances with source $s$, but the vector $\phi$ with $\phi(s)=0$ and $\phi(u)=90$ and $\phi(v)=100$ is not a $(1+\epsilon)$-approximate SSSP potential because it violates the second condition of Definition 2.7 for edge $(u, v)$ : we have $|\phi(u)-\phi(v)|=10>w(u, v)=1$.

Observation 2.8. An $\alpha$-approximate s-SSSP potential is also an $\alpha$ approximate potential for the transshipment instance with demands $\sum_{v}\left(\mathbf{1}_{v}-\mathbf{1}_{s}\right)$ (but the converse is not true).

Observation 2.9. Given a graph $G=(V, E)$ and a source s, any $\alpha$-approximate s-SSSP potential $\phi$ satisfies $|\phi(u)-\phi(v)| \leq d(u, v)$ for all $u, v \in V$.

Proof. Let $u=v_{0}, v_{1}, \ldots, v_{\ell}=v$ be the shortest path from $s$ to $v$. By property (1), we have

$$
\begin{gathered}
|\phi(u)-\phi(v)| \leq\left|\sum_{i=1}^{\ell} \phi\left(v_{i}\right)-\phi\left(v_{i-1}\right)\right| \\
\leq \sum_{i=1}^{\ell}\left|\phi\left(v_{i}\right)-\phi\left(v_{i-1}\right)\right| \leq \sum_{i=1}^{\ell} d\left(v_{i}, v_{i-1}\right)=d(u, v) .
\end{gathered}
$$

Observation 2.10. If $\phi$ is an $\alpha$-approximate s-SSSP potential, then $\phi+c \cdot 1$ is also one for any scalar $c \in \mathbb{R}$. Therefore, we can always assume w.l.o.g. that $\phi(s)=0$. In that case, by property (1), we also have $\phi(v) \geq 0$ for all $v \in V$.

Observation 2.11. Given two vectors $\phi_{1}$ and $\phi_{2}$ that satisfy property (2), the vectors $\phi_{\min }, \phi_{\max } \in \mathbb{R}^{V}$ defined as $\phi_{\min }(v):=\min \left\{\phi_{1}(v), \phi_{2}(v)\right\}$ and $\phi_{\max }(v):=\max \left\{\phi_{1}(v), \phi_{2}(v)\right\}$ for all $v \in V$ also satisfy property (2).

We now generalize the notion of SSSP potential to the case when the "source" is a subset $S \subseteq V$, not a single vertex. Essentially, the definition is equivalent to contracting all vertices in $S$ into a single source $s$, taking an $s$-SSSP potential, and setting the potential of each vertex in $S$ to the potential of $s$.
Definition 2.12 (Approximate $S$-SSSP potential). Given a graph $G=(V, E)$ and a vertex subset $S \subseteq V$, a vector $\phi \in \mathbb{R}^{V}$ is an $\alpha$ approximate $S$-SSSP potential if:

0 . For all $s \in S, \phi(s)$ takes the same value

(1) For all $v \in V$ and $s \in S, \phi(v)-\phi(s) \geq \frac{1}{\alpha} \cdot d(s, v)$

(2) For each edge $(u, v),|\phi(u)-\phi(v)| \leq w(u, v)$.

When the set $S$ is either irrelevant or clear from context, we may use $\alpha$-approximate SSSP potential (without the $S$ ) instead.

Observation 2.13. Given a graph $G=(V, E)$ and a vertex subset $S \subseteq V$, let $G^{\prime}$ be the graph with all vertices in $S$ contracted into a single vertex $s^{\prime}$. Then, if $\phi$ is an $\alpha$-approximate $S$-SSSP potential, then the vector $\phi^{\prime}$ defined as $\phi^{\prime}(v)=v$ for $v \in V \backslash S$ and $\phi^{\prime}\left(s^{\prime}\right)=\phi(s)$ for some $s \in S$ is an $\alpha$-approximate $s$-SSSP potential in $G^{\prime}$.

Also, we will need the notion of a spanner throughout the paper:

Definition 2.14 (Spanner). Given a graph $G=(V, E)$ and a parameter $\alpha \geq 1$, a subgraph $H \subseteq G$ is an $\alpha$-spanner of $G$ if for all $u, v \in V$, we have $d_{G}(u, v) \leq d_{H}(u, v) \leq \alpha d_{G}(u, v)$.

\subsection{Polynomial Aspect Ratio}

Throughout the paper, we assume that the initial input graph for the approximate SSSP problem has polynomially bounded aspect ratio, defined below:

Definition 2.15 (Aspect ratio). The aspect ratio of a graph $G=$ $(V, E)$ is the quantity $\frac{\max _{u, v \in V} d_{G}(u, v)}{\min _{u, v \in V} d_{G}(u, v)}$.

This assumption can be safely assumed: there is a reduction by Klein and Subramanian [KS92] (also used by Cohen [Coh00]) that transforms the $(1+\epsilon)$-approximate SSSP problem on a graph with arbitrary, nonnegative weights to solving $(1+\epsilon / 2)$-approximate SSSP on a collection of graphs of total size $O(m \log n)$, each with polynomially bounded aspect ratio, and requiring an additional $O(m \log n)$ work and $O(\log n)$ time. Since polynomially bounded aspect ratio is a common assumption in graph optimization problems, we will not present this reduction for sake of self-containment.

Since our SSSP algorithm is recursive, and the SSSP problem that we solve is actually the (slightly more general) SSSP potential problem, we do not apply the reduction of Klein and Subramanian again in each recursive call. Rather, we take some care to show that the aspect ratio does not blow up over recursive calls.

For the $\ell_{1}$-embedding and transshipment problems, we will handle the aspect ratio issue differently. For the $\ell_{1}$-embedding problem, we will explicitly require that the input graph has aspect ratio at most $n^{C}$ for some fixed constant $C$ (which can be made arbitrarily large). In particular, this assumption translates over in our theorem statement for parallel $\ell_{1}$-embedding (Theorem 1.3). For the transshipment problem, we will not assume that the graph has polynomial aspect ratio, but every time we recursively call transshipment, we will ensure that the demand vector has small, integral entries in the recursive instance. Assuming this guarantee on the demand vector, we reduce the transshipment problem to the case when the graph also has polynomial aspect ratio like in the SSSP case, but here, the reduction is simple enough that we include it in the paper for completeness (Lemma 3.5). 


\section{THE RECURSIVE ALGORITHM}

Our algorithm will recursively cycle through three problems: approximate SSSP potentials, approximate transshipment, and $\ell_{1}$ embedding. For the $\ell_{1}$-embedding and SSSP potential problems, we will always assume that the input graph has aspect ratio at most $n^{C}$ for some arbitrarily large but fixed constant $C>0$ (that remains unchanged throughout the recursion). The transshipment problem will require no bound on aspect ratio: we provide a simple transformation on the graph to ensure that the aspect ratio is polynomial. Let us now define the work required to solve the three problems below:

(1) $T_{\text {embed }}(m)$ is the work to $\ell_{1}$-embed a connected graph with $m$ edges and aspect ratio at most $n^{5}$ into $O(\log n)$ dimensions with distortion $O\left(\log ^{14.5} n\right)$, where the $O(\cdot)$ hides an arbitrarily large but fixed constant.

(2) $T_{\operatorname{SSSP}}(m, \epsilon)$ is the work to compute an $(1+\epsilon)$-approximate SSSP potential of a connected graph with $m$ edges and aspect ratio at most $\widetilde{O}\left(n^{5}\right)$.

(3) $T_{\mathrm{TS}}(m, \epsilon)$ is the work to compute a $(1+\epsilon)$-approximate transshipment instance of a connected graph with $m$ edges, where the demand vector $b$ is integral and satisfies $\left|b_{v}\right| \leq n-1$ for all vertices $v$.

We will not explicitly bound the (parallel) time required, but it should be clear that it is polylog $(n)$ once we bound the work below.

For space reasons, the following theorem is deferred to the full version

THEOREM 3.1. Let $G=(V, E)$ be a connected graph with $n$ vertices and $m$ edges with aspect ratio $M$, let $\beta \geq 1$ be a parameter, and let $\mathcal{A}$ be an algorithm that inputs (i) a connected graph on at most $m / \beta$ vertices and edges with aspect ratio $\widetilde{O}\left(\beta^{2} M\right)$ and (ii) a source vertex $s$, and outputs $a(1+1 / \log n)$-approximate $s$-SSSP potential. Then, there is an algorithm that computes an $\ell_{1}$-embedding of $G$ into $O(\log n)$ dimensions with distortion $O\left(\beta^{2} \log ^{6.5} n\right)$ and calls $\mathcal{A}$ at most $O\left(\log ^{2} n\right)$ times, plus $\widetilde{O}(m)$ additional work and $\operatorname{polylog}(n)$ additional time.

Corollary 3.2. $T_{\text {embed }}(m) \leq O\left(\log ^{2} n\right) \cdot T_{\mathrm{SSSP}}\left(\delta m / \log ^{4} n, 1 / \log n\right)+$ $\widetilde{O}(m)$ for any fixed, arbitrarily small constant $\delta>0$.

Proof. Apply Theorem 3.1 with $\beta:=\frac{1}{\delta} \log ^{4} n$, obtaining distortion $O\left(\beta^{2} \log ^{6.5} n\right)=O\left(\log ^{14.5} n\right)$.

The following is a corollary of our sequential transshipment result in Section 4 which constitutes our main technical contribution of the paper:

Corollary 3.3. Given an undirected graph with nonnegative weights and polynomial aspect ratio, and given an $\ell_{1}$-embedding of the graph with $\operatorname{poly} \log (n)$ distortion in $O(\log n)$ dimensions, there is a parallel algorithm to compute $a(1+\epsilon)$-approximate minimum transshipment instance in $\widetilde{O}\left(m \epsilon^{-2}\right)$ work and polylog $(n) \epsilon^{-2}$ time.

The following is Sherman's framework for the minimum transshipment problem, for which we provide a self-contained treatment through the multiplicative weights method in the full version. This is where the error boosting takes place: given a lossy polylog $(n)$ approximate $\ell_{1}$-oblivious routing algorithm encoded by the matrix $R$, we can boost the error all the way to $(1+\epsilon)$ for transshipment.
The only overhead in Sherman's framework is an additive $\widetilde{O}(m)$ work and polylog $(n)$ time (where these polylogarithmic factors depend on the approximation of the $\ell_{1}$-oblivious routing), which is ultimately what makes the recursion work out.

THeorem 3.4. Given a transshipment problem, suppose we have already computed a matrix $R$ satisfying:

(1) For all demand vectors $b \in \mathbb{R}^{n}$,

$$
\operatorname{opt}(b) \leq\|R b\|_{1} \leq \kappa \cdot \operatorname{opt}(b)
$$

(2) Matrix-vector products with $R$ and $R^{T}$ can be computed in $M$ work and $\operatorname{polylog}(n)$ time

Then, for any transshipment instance with demand vector $b$, we can compute a flow vector $f$ and a vector of potentials $\tilde{\phi}$ in $\widetilde{O}\left(\kappa^{2}(m+n+\right.$ M) $\left.\epsilon^{-2}\right)$ time that satisfies:

(1) $\|C f\|_{1} \leq(1+\epsilon) b^{T} \tilde{\phi} \leq(1+\epsilon) \operatorname{opt}(b)$

(2) $\operatorname{opt}(A f-b) \leq \beta \operatorname{opt}(b)$

Lastly, there is one minor mismatch: Corollary 3.3 assumes that the graph has polynomial aspect ratio, while the problem for $T_{\mathrm{TS}}(\cdot)$ does not assume such a thing, but rather assumes that the demand vector has entries restricted to $\{-(n-1),-(n-2), \ldots, n-2, n-1\}$. It turns out that given this restriction on the demand vector, the polynomial aspect ratio of the graph can be obtained for free. This is proved in the full version.

Lemma 3.5. Given a transshipment instance with graph $G=(V, E)$ with $n$ vertices and $m$ edges and an integer demand vector $b$ satisfying $\left|b_{v}\right| \leq M$ for all $v \in V$, we can transform $G$ into another graph $\widehat{G}$ on $n$ vertices and at most $m$ edges such that $\widehat{G}$ has aspect ratio at most $n^{4} M$, and $\operatorname{opt}_{G}(b) \leq \operatorname{opt}_{\widehat{G}}(b) \leq\left(1+1 / n^{2}\right)$ opt $_{G}(b)$. The transformation takes $\widetilde{O}(m)$ work and $\operatorname{polylog}(n)$ time.

Corollary 3.6. $T_{\mathrm{TS}}(m, \epsilon) \leq T_{\text {embed }}(m)+\widetilde{O}\left(m / \epsilon^{2}\right)$. That is, outside of an $\ell_{1}$-embedding into $O(\log n)$ dimensions with distortion $O\left(\log ^{14.5} n\right)$, the additional work to compute $(1+\epsilon)$-approximate transshipment is $\widetilde{O}\left(m / \epsilon^{2}\right)$. Moreover, the additional time required is $\widetilde{O}\left(1 / \epsilon^{2}\right)$.

Proof. By assumption, the demand vector $b_{v}$ is integral and satisfies $\left|b_{v}\right| \leq n-1$ for all vertices $v$. Apply Lemma 3.5 with $M:=n-1$ so that the aspect ratio of the modified graph $\widehat{G}$ is at most $n^{5}$, which is polynomial, and the optimal solution changes by factor at most $\left(1+1 / n^{2}\right)$. Compute an $\ell_{1}$-embedding of $\widehat{G}$ into polylog $(n)$ dimensions (in $T_{\text {embed }}(m)$ work), and then apply Corollary 3.3 with approximation factor $(1+\epsilon / 2)$. The final approximation factor is $\left(1+1 / n^{2}\right)(1+\epsilon / 2)$, which is at most $(1+\epsilon)$ for $\epsilon \geq \Omega\left(1 / n^{2}\right)$. (If $\epsilon=O\left(1 / n^{2}\right)$, then an algorithm running in time $\widetilde{O}\left(1 / \epsilon^{2}\right) \geq \widetilde{O}\left(n^{4}\right)$ is trivial.)

We now present the reduction from approximate SSSP to approximate transshipment, partially inspired by a similar routine in [BKKL16]; for completeness, we give a self-contained proof of the reduction in the full version in the form of this theorem:

Theorem 3.7. Let $G=(V, E)$ be a graph with $n$ vertices and $m$ edges, and let $\epsilon>0$ be a parameter. Let $\mathcal{A}$ be an algorithm that inputs any graph with at most $n$ vertices and $m$ edges and an integral demand vector $b \in \mathbb{R}^{V}$ satisfying $\left|b_{v}\right| \leq n-1$ for all $v \in V$, and outputs $a$ 
$\left(1+\Theta\left(\frac{\epsilon}{\log n}\right)\right)$-approximate flow-potential pair to the transshipment instance of that graph with demands $b$. Then, for any source $s \in V$, we can compute a $(1+\epsilon)$-approximate SSSP tree with source $s$ in $O\left(\log ^{2} n\right)$ calls to $\mathcal{A}$, plus an additional $\widetilde{O}(m)$ work and $\operatorname{polyl} \log (n)$ time.

Corollary 3.8. $T_{\mathrm{SSSP}}(m, \epsilon) \leq O\left(\log ^{2} n\right) \cdot T_{\mathrm{TS}}\left(m, \Theta\left(\frac{\epsilon}{\log n}\right)\right)+\widetilde{O}(m)$.

Proof. This is essentially Theorem 3.7 in recursive form.

Corollary 3.9. $T_{\mathrm{SSSP}}(m, \epsilon) \leq O\left(\log ^{4} n\right) \cdot T_{\mathrm{SSSP}}\left(\delta m / \log ^{4} n, 1 / \log n\right)+$ $\widetilde{O}\left(m / \epsilon^{2}\right)$ for any fixed, arbitrarily small constant $\delta>0$.

Proof. Follows directly from Corollaries 3.2, 3.6 and 3.8.

Corollary 3.10. Given any connected graph $G=(V, E)$ with $n$ vertices and $m$ edges, for any source $s \in V$, we can compute $a(1+\epsilon)$ approximate s-SSSP potential in $\widetilde{O}\left(m / \epsilon^{2}\right)$ work and $\widetilde{O}\left(1 / \epsilon^{2}\right)$ time.

Proof. The work bound follows by Corollary 3.9. For the time bound, observe that in the recursion of Corollary 3.9, by setting $\delta>0$ small enough, the total graph size $O\left(\log ^{4} n\right) \cdot \delta m / \log ^{4} n \leq m / 2$ drops by at least half on each recursion level. Therefore, the total work is dominated by the work at the root of the recursion tree, which is $\widetilde{O}\left(m / \epsilon^{2}\right)$. The recursion has $O(\log n)$ levels, and on each level, all instances can be executed in parallel, taking $\widetilde{O}\left(1 / \epsilon^{2}\right)$ time by Corollary 3.6, so the time bound follows.

\section{1 $\ell_{1}$-Embedding from Approximate SSSP Potential}

In this section, we briefly overview our $\ell_{1}$-embedding algorithm, which is necessary for Theorem 3.1 and hence, the reduction from $\ell_{1}$-embedding to smaller instances of approximate SSSP potentials. Our $\ell_{1}$-embedding algorithm is very similar to the one of [LLR95], except utilizing approximate SSSP instead of exact, as well as slightly simplified at the expense of several logarithmic factors. Due to its similarily, we defer its proof to the full version.

Theorem 3.11. Let $G=(V, E)$ be a graph with $n$ vertices and $m$ edges, and let $\mathcal{A}$ be an algorithm that inputs any vertex set $S \subseteq V$ and outputs $a(1+1 / \log n)$-approximate $S$-SSSP potential of $G$. Then, there is an algorithm that computes an $\ell_{1}$-embedding of $G$ into $O(\log n)$ dimensions with distortion $O\left(\log ^{4.5} n\right)$ and calls $\mathcal{A}$ at most $O\left(\log ^{2} n\right)$ times, plus $\widetilde{O}(m)$ additional work and $\operatorname{polylog}(n)$ additional time.

We will focus our attention on a slightly different variant which we show implies Theorem 3.11:

Lemma 3.12. Let $G=(V, E)$ be a graph with $n$ vertices and $m$ edges, and let $\mathcal{A}$ be an algorithm that inputs any vertex set $S \subseteq V$ and outputs $a(1+1 / \log n)$-approximate $S$-SSSP potential of $G$. Then, there is an algorithm that computes an $\ell_{1}$-embedding of $G$ into $O\left(\log ^{2} n\right)$ dimensions with distortion $O\left(\log ^{3} n\right)$ and calls $\mathcal{A}$ at most $O\left(\log ^{2} n\right)$ times, plus $\widetilde{O}(m)$ additional work and $\operatorname{polylog}(n)$ additional time.

Lemma 3.12 is proved in the full version. We now show that Lemma 3.12 implies Theorem 3.11. Since the $\ell_{1}$ and $\ell_{2}$ metrics are at most a multiplicative $\sqrt{k}$ factor apart in dimension $k$, the embedding of Lemma 3.12 has distortion $O\left(\log ^{3} n\right) \cdot \sqrt{O\left(\log ^{2} n\right)}=O\left(\log ^{4} n\right)$ in the $\ell_{2}$ metric. Next, apply Johnson-Lindenstrauss dimensionality reduction [JL84] on this set of vectors, reducing the dimension to $O(\log n)$ with a constant factor increase in the distortion. We now move back to the $\ell_{1}$ metric, incurring another $O(\sqrt{\log n})$ factor in the distortion, for a total of $O\left(\log ^{4.5} n\right)$ distortion.

\subsection{Sparsification and Recursion to Smaller Instances}

In this section, we briefly overview the main ideas behind our sparsification process in order to reduce the $\ell_{1}$-embedding problem to approximate SSSP instances of sufficiently smaller size:

Theorem 3.1. Let $G=(V, E)$ be a connected graph with $n$ vertices and $m$ edges with aspect ratio $M$, let $\beta \geq 1$ be a parameter, and let $\mathcal{A}$ be an algorithm that inputs (i) a connected graph on at most $m / \beta$ vertices and edges with aspect ratio $\widetilde{O}\left(\beta^{2} M\right)$ and (ii) a source vertex $s$, and outputs $a(1+1 / \log n)$-approximate $s$-SSSP potential. Then, there is an algorithm that computes an $\ell_{1}$-embedding of $G$ into $O(\log n)$ dimensions with distortion $O\left(\beta^{2} \log ^{6.5} n\right)$ and calls $\mathcal{A}$ at most $O\left(\log ^{2} n\right)$ times, plus $\widetilde{O}(m)$ additional work and $\operatorname{polylog}(n)$ additional time.

One key tool we will use is the concept of ultra-sparse spanners, introduced by Elkin and Neiman [KMP14a]. Here, we will rename them to ultra-spanners to further emphasize their connection to ultra-sparsifiers in [KMP14b, Pen16]. These are spanners that are so sparse that they are almost "tree-like" when the graph is sparse enough: a graph with $(n-1)+t$ edges for some small $t$ (say, $t=m / \operatorname{polylog}(n)$ ). We will utilize the following ultra-spanner construction, which is adapted from the one of [MPVX13]; while theirs is not ultra-sparse, we modify it to be, at the expense of an additional $k$ factor in the stretch. The ultra-spanner algorithm is deferred to the full version.

Lemma 3.13. Given a weighted graph $G$ with polynomial aspect ratio and a parameter $k \geq \Omega(1)$, there is an algorithm to compute a $k^{2}$-spanner of $G$ with $(n-1)+O\left(\frac{m \log n}{k}\right)$ edges in $\widetilde{O}(m)$ work and polylog(n) time.

Why are ultra-spanners useful for us? Their key property, stated in the lemma below, is that we can compute an $\alpha$-approximate SSSP potential on an ultra-spanner by recursively calling $\alpha$-approximate SSSP potentials on a graph with potentially much fewer vertices. To develop some intuition on why this is possible, observe first that if a connected graph has $(n-1)$ edges, then it is a tree, and SSSP is very easy to solve on trees. If the graph has $(n-1)+t$ edges instead for some small value of $t$, then the graph is almost "tree-like" outside of at most $2 t$ vertices: take an arbitrary spanning tree, and let these vertices be the endpoints of the $t$ edges not on the spanning tree. We want to say that the graph is "easy" outside a graph on $2 t$ vertices, so that we can solve a SSSP problem on the "hard" part of size $O(t)$ and then extend the solution to the rest of the graph in an efficient manner. This is indeed our approach, and it models closely off the concept of a $j$-tree by Madry [Mad10], which is also used in Peng's recursive maximum flow algorithm [Pen16].

This recursion idea can be considered a vertex-sparsification step, following the edge-sparsification that the ultra-spanner achieves. We package the vertex-sparsification in the lemma below; while this lemma works for all $t$, the reader should imagine that $t=$ 
$m / \operatorname{polylog}(n)$, since that is the regime where the lemma will be applied. Due to its length and technical involvement, the proof is deferred to the full version.

Lemma 3.14. Let $G=(V, E)$ be a connected graph with aspect ratio $M$ with $n$ vertices and $(n-1)+t$ edges, and let $\alpha>0$ be a parameter. Let $\mathcal{A}$ be an algorithm that inputs a connected graph on at most $70 t$ vertices and edges and aspect ratio $\widetilde{O}(M)$ and outputs an $\alpha$-approximate $s$-SSSP potential of that graph. Then, for any subset $S \subseteq V$, we can compute an $\alpha$-approximate $S$-SSSP potential of $G$ through a single call to $\mathcal{A}$, plus $\widetilde{O}(m)$ additional work and $\operatorname{polylog}(n)$ additional time.

We now prove Theorem 3.1 assuming Lemma 3.14:

Proof (Theorem 3.1). Invoke Lemma 3.13 with $k:=C \beta \log n$ for a large enough constant $C>0$, producing a spanner $H$ with $(n-$ 1) $+O\left(\frac{m \log n}{k}\right)$ edges and stretch at most $k^{2}=O\left(\beta^{2} \log ^{2} n\right)$. Since $H$ is a spanner, we have $\min _{u, v \in V} d_{H}(u, v) \geq \min _{u, v \in V} d_{G}(u, v)$ and $\max _{u, v \in V} d_{H}(u, v) \leq k^{2} \max _{u, v \in V} d_{G}(u, v)$, so $H$ has aspect ratio $\widetilde{O}\left(\beta^{2} M\right)$. Since $G$ is connected, we have $O(m \log n / k) \leq m /(70 \beta)$ for $C$ large enough, so $H$ has at most $(n-1)+m /(70 \beta)$ edges. Then, apply Lemma 3.14 on $H$ with $t:=m /(70 \beta), \alpha:=1+1 / \log n$, and the algorithm $\mathcal{A}$, producing an algorithm $\mathcal{A}_{H}$ that inputs any vertex set $S \subseteq V$ and outputs an $(1+1 / \log n)$-approximate $S$-SSSP potential on $H$ through a single call to $\mathcal{A}$, plus $\widetilde{O}(m)$ additional work and polylog $(n)$ additional time.

Next, apply Theorem 3.11 on the spanner $H$ with algorithm $\mathcal{A}_{H}$, embedding $H$ into $O(\log n)$ dimensions with distortion $O\left(\log ^{4.5} n\right)$ through $O\left(\log ^{2} n\right)$ calls to $\mathcal{A}_{H}$, which in turn makes $O\left(\log ^{2} n\right)$ calls to $\mathcal{A}$; the additional work and time remain $\widetilde{O}(m)$ and polylog$(n)$, respectively.

Finally, since $H$ is a spanner for $G$ with stretch $O\left(\beta^{2} \log ^{2} n\right)$, the $\ell_{1}$-embedding of $H$ with stretch $O\left(\log ^{4.5} n\right)$ is automatically an $\ell_{1}$-embedding of $G$ with distortion $O\left(\beta^{2} \log ^{2} n\right) \cdot O\left(\log ^{4.5} n\right)=$ $O\left(\beta^{2} \log ^{6.5} n\right)$.

\section{$4 \ell_{1}$-OBLIVIOUS ROUTING AND SEQUENTIAL TRANSSHIPMENT}

This section is dedicated to the sequential transshipment result (Theorem 1.4, restated below) and constitutes our main technical contribution of the paper.

Theorem 1.4 (Sequential transshipment). There is an algorithm that, given an undirected graph with nonnegative weights and polynomial aspect ratio, computes a $(1+\epsilon)$-approximation to minimum transshipment in time $O\left(\left(m \log ^{10} n+n \log ^{15} n\right) \epsilon^{-2}(\log \log n)^{O(1)}\right)$.

Throughout the section, we make no references to parallel algorithms, keeping all our algorithms entirely sequential in an effort to focus solely on Theorem 1.4. Nevertheless, to a reader with parallel algorithms in mind, it should be clear that all algorithms in this section can be parallelized to require polylog $(n)$ parallel time. To streamline the transition to parallel algorithms in the rest of the paper, we package a parallel version of the main routine in this section in an easy-to-use statement, Corollary 3.3.

\subsection{Improved $\ell_{1}$-Oblivious Routing: Our Techniques}

The key technical ingredient in our transshipment algorithm is an improved $\ell_{1}$-oblivious routing, scheme. Our algorithm begins similarly to Sherman's [She17b]: compute an $\ell_{1}$-embedding into low dimensions at a small loss in approximation. Sherman chooses dimension $O(\sqrt{\log n})$ and loses a $2^{O(\sqrt{\log n})}$ factor in the distortion, and then constructs an oblivious routing in the embedded space in time exponential in the dimension. Our oblivious routing is polynomial in the dimension, so we can afford to choose dimension $O(\log n)$, giving us polylog $(n)$ distortion. The benefit in the $\ell_{1}$-embedding is that we now have a nice geometric property of the vertices, which are now points in $O(\log n)$-dimensional space under the $\ell_{1}$ metric.

In the full version, we provide some intuition for the oblivious routing algorithm and our construction, but for space reasons, it is omitted here.

\subsection{Sherman's Framework}

Below, we state a paraphrased version of Sherman's framework [She17b]. For the simplest reference, see Corollary 1 and Lemma 4 of [KNP19]. We also provide a proof via multiplicative weights in the full version, whose running time suffers an additional factor of $\log (n / \epsilon)$ due to a binary search overhead. For Theorem 1.4, we will use the theorem below, while for the parallel algorithms, the weaker Theorem 3.4 suffices.

Theorem 4.1 (Sherman, Paraphrased). Given a transshipment problem, suppose we have already computed a matrix $R$ satisfying:

(1) For all demand vectors $b \in \mathbb{R}^{n}$, opt $(b) \leq\|R b\|_{1} \leq \kappa \cdot \operatorname{opt}(b)$

(2) Matrix-vector products with $R$ and $R^{T}$ can be computed in $M$ time

Then, for any transshipment instance with demand vector $b$, we can compute a flow vector $\tilde{f}$ and a vector of potentials $\tilde{\phi}$ in $O\left(\kappa^{2}(m+n+\right.$ M) $\left.\log (m)\left(\epsilon^{-2}+\log (1 / \beta)\right)\right)$ sequential time that satisfies:

(1) $\|C \tilde{f}\|_{1} \leq(1+\epsilon) b^{T} \tilde{\phi} \leq(1+\epsilon) \operatorname{opt}(b)$

(2) $\operatorname{opt}(A \tilde{f}-b) \leq \beta \operatorname{opt}(b)$

The matrix $R$ encodes the oblivious routing algorithm. Also, intuitively, the more efficient the oblivious routing, the sparser the matrix $R$, although this relation is not as well-defined. Nevertheless, there is an equivalence between oblivious routing schemes and matrices $R$ that satisfy requirement (1) of Theorem 4.1. But since Sherman's framework uses steepest descent methods that involve matrix algebra, a matrix $R$ with efficient matrix-vector multiplications is most convenient for the framework.

Our main technical result is computing such a matrix $R$ efficiently:

Theorem 4.2 (Computing $R$ ). Given a transshipment problem, we can compute a matrix $R$ with $O\left(n \log ^{5} n(\log \log n)^{O(1)}\right)$ nonzero entries, such that for any demand vector $b$,

$$
\operatorname{opt}(b) \leq\|R b\|_{1} \leq O\left(\log ^{4.5} n\right) \cdot \operatorname{opt}(b) .
$$

The algorithm succeeds w.h.p., and runs in $O\left(m \log ^{2} n+n \log ^{10}(\log \log n)^{O(1)}\right)$ sequential time. 
With this fast routing algorithm in hand, our main theorem, restated below, follows immediately. Our proof uses low-stretch spanning trees [AN12], so for a self-contained rendition, we remark after the proof that low-stretch spanning trees can be removed at the expense of another $\log n$ factor.

Theorem 1.4 (Sequential transshipment). There is an algorithm that, given an undirected graph with nonnegative weights and polynomial aspect ratio, computes a $(1+\epsilon)$-approximation to minimum transshipment in time $O\left(\left(m \log ^{10} n+n \log ^{15} n\right) \epsilon^{-2}(\log \log n)^{O(1)}\right)$.

Proof. Apply Theorem 4.1 with the parameters $\kappa:=O\left(\log ^{4.5} n\right)$ and $M:=O\left(n \log ^{5} n(\log \log n)^{O(1)}\right)$ guaranteed by Theorem 4.2, along with $\beta:=\Theta(\epsilon /(\log n \log \log n))$. This takes time

$$
\begin{gathered}
O\left(\log ^{9} n \cdot\left(m+n \log ^{5} n\right) \cdot \log n \cdot \epsilon^{-2} \cdot(\log \log n)^{O(1)}\right) \\
\quad=O\left(\left(m \log ^{10} n+n \log ^{15} n\right) \cdot \epsilon^{-2} \cdot(\log \log n)^{O(1)}\right),
\end{gathered}
$$

and outputs a flow $\tilde{f}$ with $\|C f\|_{1} \leq(1+\epsilon) \operatorname{opt}(b)$ and opt $(A \tilde{f}-b) \leq$ $\beta$ opt $(b)$.

To route the remaining demand $A \tilde{f}-b$, for $O(\log n)$ independent iterations, compute a low-stretch spanning tree in $O(n \log n \log \log n)$ time with expected stretch $O(\log n \log \log n)$ [AN12] and solve (exact) transshipment in linear time on the tree. In each iteration, the expected cost is at most $O(\log n \log \log n) \cdot \beta \operatorname{opt}(b)=\epsilon \operatorname{opt}(b)$ for an appropriate choice of $\beta$, so w.h.p., one iteration has cost at most twice the expectation. Let $f^{\prime}$ be this flow, which satisfies $\|C f\|_{1} \leq 2 \epsilon \operatorname{opt}(b)$ and $A f^{\prime}=A \tilde{f}-b$. The composed flow $\tilde{f}-f^{\prime}$ is our final flow, which satisfies $\left\|C\left(f-f^{\prime}\right)\right\|_{1} \leq\|C f\|_{1}+\left\|C f^{\prime}\right\|_{1} \leq$ $(1+3 \epsilon) \operatorname{opt}(b)$ and $A\left(\tilde{f}-f^{\prime}\right)=b$. Finally, to obtain a $(1+\epsilon)-$ approximation, we can simply reset $\epsilon \leftarrow \epsilon / 3$.

Remark 4.3. To eliminate the use of low-stretch spanning trees, we can set $\beta:=\epsilon / n$ instead, picking up another $\log n$ factor in Theorem 4.1. Then, we can route the remaining demand along a minimum spanning tree, which is an $(n-1)$-approximation of optimum, or at most $(n-1) \beta \operatorname{opt}(b) \leq \epsilon \operatorname{opt}(b)$.

\subsection{Polynomial Aspect Ratio}

Throughout this section, we assume that the input graph has polynomial aspect ratio, since that is assumed in Theorem 1.4.

\subsection{Reduction to $\ell_{1}$ Metric}

The reduction to the $\ell_{1}$ metric is standard, via Bourgain's embedding:

Definition 4.4. For $p \geq 1$, an $\ell_{p}$-embedding of a graph $G=(V, E)$ with distortion $\alpha$ and dimension $k$ is a collection of vectors $\left\{x_{v} \in\right.$ $\left.\mathbb{R}^{k}: v \in V\right\}$ such that

$$
\forall u, v \in V: d_{G}(u, v) \leq\left\|x_{u}-x_{v}\right\|_{p} \leq \alpha d_{G}(u, v) .
$$

Theorem 4.5 (FAst Bourgain's embedding). Given a graph with $m$ edges, there is a randomized $O\left(m \log ^{2} n\right)$ time algorithm that computes an $\ell_{1}$-embedding of the graph with distortion $O\left(\log ^{1.5} n\right)$ and dimension $O(\log n)$.
Proof (Sкетсн). Apply the fast embedding algorithm of [LLR95] in $\ell_{2}$, which runs in $O\left(m \log ^{2} n\right)$ randomized time and w.h.p., computes an $\ell_{2}$-embedding of the graph with distortion $O(\log n)$ and dimension $O\left(\log ^{2} n\right)$. Next, apply Johnson-Lindenstrauss dimensionality reduction [JL84] on this set of vectors, reducing the dimension to $O(\log n)$ with a constant factor increase in the distortion. Lastly, since the $\ell_{1}$ and $\ell_{2}$ metrics are at most a multiplicative $\sqrt{k}$ factor apart in dimension $k$, this same set of vectors in $O(\log n)$ dimensions has distortion $O\left(\log ^{1.5} n\right)$ in the $\ell_{1}$ metric.

Finally, since our input graph is assumed to have polynomial aspect ratio, so do the embedded points under the $\ell_{1}$ metric. In particular, suppose that before applying Theorem 4.5 we scaled the graph $G$ so that the smallest edge had length 1 . Then, the embedding satisfies the following:

Assumption 4.6 (Polynomial aspect ratio). For some constant $c>$ 0 , the vectors $\left\{x_{v}: v \in V\right\}$ satisfy $1 \leq d(u, v) \leq n^{c}$ for all $u, v \in V$.

\subsection{Oblivious Routing on $\ell_{1}$ Metric}

In this section, we work under the $\ell_{1}$ metric in $O(\log n)$ dimensions (the setting established by Theorem 4.5) with the additional Assumption 4.6. Our main technical result is:

THeOrem 4.7. We can compute a matrix $R$ with $O\left(n \log ^{5} n(\log \log n)^{O(1)}\right)$ nonzero entries, such that for any demand vector $b$,

$$
\operatorname{opt}(b) \leq\|R b\|_{1} \leq O\left(\log ^{3} n\right) \cdot \operatorname{opt}(b) .
$$

The algorithm succeeds w.h.p., and runs in $O\left(n \log ^{10}(\log \log n)^{O(1)}\right)$ sequential time.

Together with the $O(\log n)$ additional distortion from Theorem 4.5, this proves Theorem 4.2.

Before we begin with the algorithm, we first make a reduction from "w.h.p., for all $b$ " to the weaker statement "for each $b$, w.h.p.". The former requires that w.h.p., the statement holds for every demand vector $b$, while the latter requires that for any given demand vector $b$, the statement holds w.h.p. (Since there are uncountably many such $b$, the latter does not imply the former in general.) This simplifies our argument, since we only need to focus on a given demand vector $b$, which will often be fixed throughout a section. Before we state and prove the reduction, for each $v \in V$, let us define $\chi_{v}: V \rightarrow \mathbb{R}$ as the function that is 1 at $v$ and 0 elsewhere.

Lemma 4.8. Suppose a randomized algorithm outputs a matrix $R$ such that for any given demand vector $b$, we have opt $(b) \leq\|R b\|_{1}$ with probability 1 , and $\|R b\|_{1} \leq \kappa \cdot \operatorname{opt}(b)$ w.h.p. Then, this same matrix $R$ satisfies the following stronger property: w.h.p., for any demand vector $b$, we have opt $(b) \leq\|R b\|_{1} \leq \kappa \cdot \operatorname{opt}(b)$.

Proof. W.h.p., the matrix $R$ satisfies $\left\|R\left(\chi_{u}-\chi_{v}\right)\right\|_{1} \leq \kappa \cdot \operatorname{opt}\left(\chi_{u}-\right.$ $\left.\chi_{v}\right)$ for each of the $O\left(n^{2}\right)$ demand vectors $\chi_{u}-\chi_{v}(u, v \in V)$. We claim that in this case, $R$ actually satisfies opt $(b) \leq\|R b\|_{1} \leq$ $\kappa \cdot \operatorname{opt}(b)$ for all demand vectors $b$.

Fix any demand vector $b$, and suppose that the flow achieving $\operatorname{opt}(b)$ routes $f(u, v) \geq 0$ flow from $u$ to $v$ for each $u, v \in \mathbb{R}^{k}$, so that $b=\sum_{u, v} f(u, v) \cdot\left(\chi_{u}-\chi_{v}\right)$ and $\operatorname{opt}(b)=\sum_{u, v} f(u, v) \cdot\|u-v\|_{1}$. 
Then, we still have opt $(b) \leq\|R b\|_{1}$ by assumption, and for the other direction, we have

$$
\begin{gathered}
\|R b\|_{1}=\left\|R \cdot \sum_{u, v} f(u, v)\left(\chi_{u}-\chi_{v}\right)\right\|_{1} \leq \sum_{u, v} f(u, v)\left\|R\left(\chi_{u}-\chi_{v}\right)\right\|_{1} \\
\leq \kappa \cdot \sum_{u, v} f(u, v) \operatorname{opt}\left(\chi_{u}-\chi_{v}\right)=\kappa \cdot \operatorname{opt}(b),
\end{gathered}
$$

as desired.

We also introduce a specific formulation of a routing that helps in the analysis of our algorithm:

Definition 4.9 (Routing). Given a metric space $(V, d)$, a routing is a function $R: V \times V \rightarrow \mathbb{R}$ such that

$$
\forall u, v \in V: R(u, v)=-R(v, u)
$$

A routing $R$ satisfies demand vector $b \in \mathbb{R}^{V}$ if

$$
\forall v \in V: \sum_{u \in V} R(u, v)=b_{v}
$$

A routing $R$ has cost

$$
\operatorname{cost}(R):=\sum_{u, v \in V}|R(u, v)| \cdot\|u-v\|_{1},
$$

and is optimal for demand vector $b$ if it minimizes $\operatorname{cost}(R)$ over all routings $R^{\prime}$ satisfying $b$.

For example, if $b=\chi_{u}-\chi_{v}$ for some $u, v \in V$, then one feasible routing (in fact, the optimal one) is $R(u, v)=1, R(v, u)=-1$, and $R(x, y)=0$ for all other pairs $(x, y)$, which has cost $2\|u-v\|_{1}$.

Note that $\operatorname{cost}(R)$ is actually twice the value of the actual transshipment cost in the $\ell_{1}$ metric. However, since this notion of routing is only relevant in our analysis, and we are suffering a polylog $(n)$ approximation anyway, we keep it this way for future simplicity.

Observation 4.10. Given a metric space $(V, d)$ and demand vector $b \in \mathbb{R}^{V}$, opt $(b)$ is equal to the minimum value of $\frac{1}{2} \operatorname{cost}(R)$ over all routings $R$ that satisfy demand vector $b$.

We first introduce our algorithm in pseudocode below, along with the following notations. For real numbers $x$ and $W>0$, define $\lfloor x\rfloor_{W}:=\lfloor x / W\rfloor \cdot W$ as the greatest (integer) multiple of $W$ less than or equal to $W$ (so that if $W=1$, then $\lfloor x\rfloor_{W}=\lfloor x\rfloor$ ), and similarly, define $\lceil x\rceil_{W}:=\lceil x / W\rceil \cdot W$ as the smallest (integer) multiple of $W$ greater than or equal to $W$.

The lines marked imaginary are actually not executed by the algorithm. They are present to define the "imaginary" routings $R_{t}^{*}$, which exist only for our analysis. We could have defined the $R_{t}^{*}$ separately from the algorithm, but we decided that including them alongside the algorithm is more concise and (more importantly) illustrative.

Lastly, we remark that the algorithm does not require the input $b \in \mathbb{R}^{V}$ to be a demand vector. This observation is important when building the matrix $R$, where we will evaluate the algorithm on only the vectors $\chi_{v}$ for $v \in V$, which are not demand vectors.

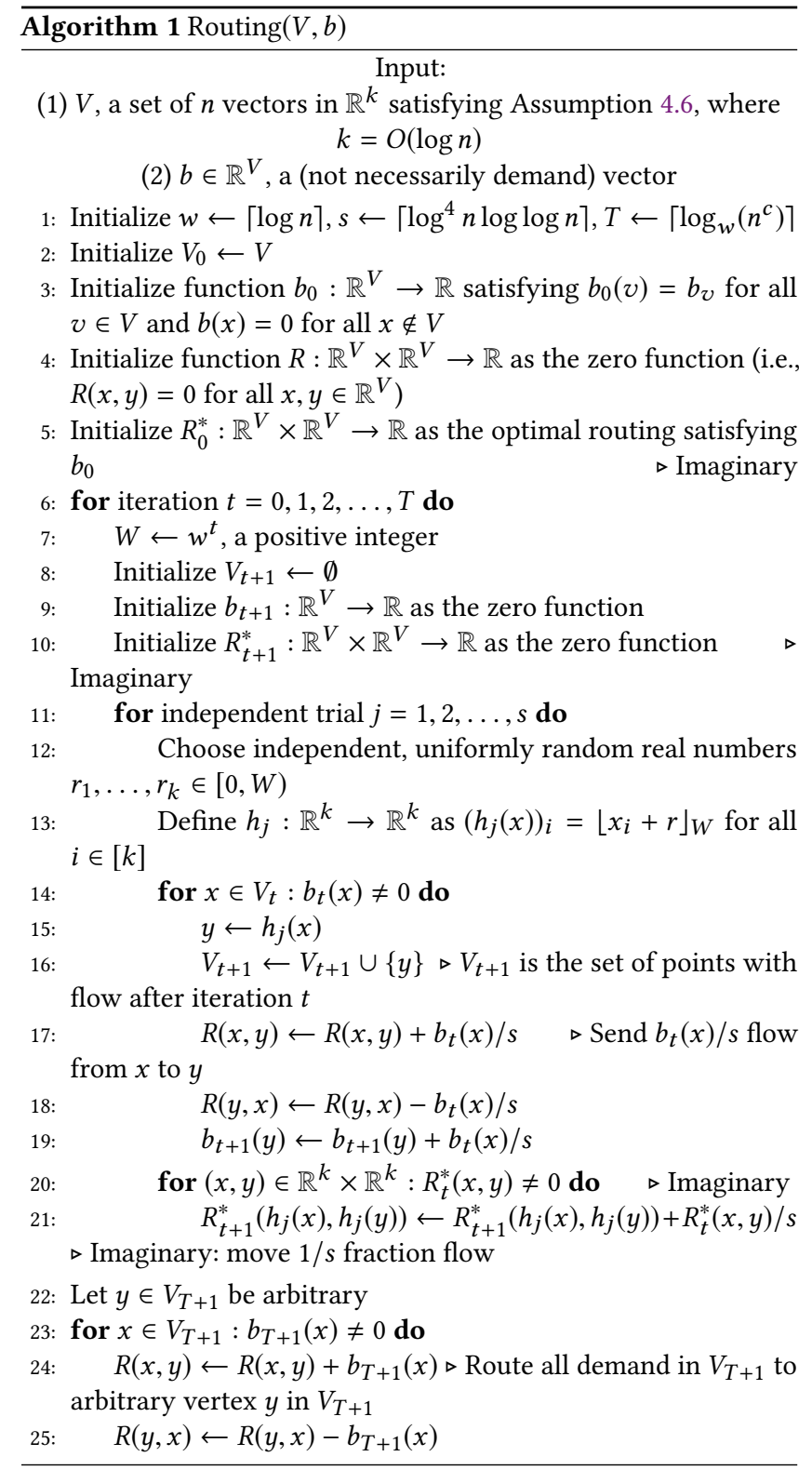

4.5.1 Proof Outline. The purpose of the "imaginary" routing $R_{t}^{*}$ is to upper bound our actual cost. For $R_{t}^{*}$ to be a reasonable upper bound, it should not increase too much over the iterations. These properties are captured in the two lemmas below, proved in Section 4.5.2:

Lemma 4.11. The total cost of routing on each iteration $t$ (lines 17 and 18) is at most $k w \cdot \operatorname{cost}\left(R_{t}^{*}\right)$.

Lemma 4.12. With probability $1-n^{-\omega(1)}, \operatorname{cost}\left(R_{t+1}^{*}\right) \leq(1+$ $\left.\frac{1}{\log n}\right) \operatorname{cost}\left(R_{t}^{*}\right)$ for each iteration $t$.

The last routing on lines 24 and 25 is handled in Section 4.5.4:

Lemma 4.13. The total cost of routing on lines 24 and 25 is at most $O(k w) \cdot \operatorname{cost}\left(R_{T+1}^{*}\right)$. 
The three lemmas above imply the following corollary:

Corollary 4.14. With probability $1-n^{-\omega(1)}$, the total cost of routing in the algorithm is at most $O(k w T) \cdot \operatorname{opt}(b)$.

Proof. By applying Lemma 4.12 inductively over all $t$, with probability $1-n^{-\omega(1)}$,

$$
\begin{gathered}
\operatorname{cost}\left(R_{t+1}^{*}\right) \leq\left(1+\frac{1}{\log n}\right)^{t} \operatorname{cost}\left(R_{0}^{*}\right)=\left(1+\frac{1}{\log n}\right)^{t} \operatorname{opt}(b) \\
\leq\left(1+\frac{1}{\log n}\right)^{O(\log n)} \operatorname{opt}(b)=O(1) \cdot \operatorname{opt}(b) .
\end{gathered}
$$

By Lemma 4.11, the cost of routing on iteration $t$ is at most $k w$. $\operatorname{cost}\left(R_{t}^{*}\right) \leq O(k w) \cdot \operatorname{opt}(b)$. Summing over all $t$, we obtain a total cost of $O(k w T) \cdot \operatorname{opt}(b)$ over iterations 0 through $T$. Finally, by Lemma 4.13, the cost of routing on lines 24 and 25 is at most $O(k w)$. $\operatorname{cost}\left(R_{T+1}^{*}\right) \leq O(k w) \cdot o p t(b)$ as well.

At the same time, the routing should be "sparse", to allow for a near-linear time algorithm. Our sparsity is captured by the following lemma, proved in Section 4.5.3:

Lemma 4.15. For each $\chi_{v}$, if we run Algorithm 1 on demands $\chi_{v}$, every function $b_{t}$ has $O(s)$ nonzero values in expectation. Moreover, each function $b_{t}$ can be computed in $O\left(s^{2}\right)$ expected time.

This sparsity guarantee ensures that the matrix $R$ that we compute is also sparse, specified in the lemma below, proved in Section 4.5.5:

Lemma 4.16. We can compute a matrix $R$ such that $\|R b\|_{1}$ approximates the cost of routing in Algorithm 1 to factor $O(1)$, and $R$ has $O(s T n)=O\left(n \log ^{5} n(\log \log n)^{O(1)}\right)$ nonzero entries. The algorithm succeeds w.h.p., and runs in time $O\left(s^{2} T n \log n\right)=O\left(n \log ^{10} n(\log \log n)^{O(1)}\right)$.

Finally, with Corollary 4.14 and Lemmas 4.15 and 4.16, we prove our main result below:

Theorem 4.7. We can compute a matrix $R$ with $O\left(n \log ^{5} n(\log \log n)^{O(1)}\right)$ nonzero entries, such that for any demand vector $b$,

$$
\operatorname{opt}(b) \leq\|R b\|_{1} \leq O\left(\log ^{3} n\right) \cdot \operatorname{opt}(b) .
$$

The algorithm succeeds w.h.p., and runs in $O\left(n \log ^{10}(\log \log n)^{O(1)}\right)$ sequential time.

Proof. By Lemma 4.16, we can compute a matrix $R$ that approximates the cost of routing in Algorithm 1 to factor $O(1)$. By Corollary 4.14, this cost of routing is at most $O(k w T) \cdot \operatorname{opt}(b)$, and it is clearly at least $\operatorname{opt}(b)$. Thus, $R$ approximates opt $(b)$ by an $O(k w T)=O\left(\log ^{3} n\right)$ factor. The requirements on $R$ are guaranteed by Lemma 4.16 .

4.5.2 Proof of Approximation. We first begin with a few invariants of Algorithm 1, whose proofs are trivial by inspection:

Invariant 4.17. At the end of iteration $t, R$ satisfies demand vector $b_{t+1}$.
Proof. Suppose by induction on $t$ that $R$ satisfies demand vector $b_{t}$ at the beginning of iteration $t$. Recall that for $R$ to satisfy $b_{t+1}$ at the end of iteration $t$, we must have $\sum_{u} R(u, v)=b_{t+1}(v)$ for all $v$ by then. For each $v$, we track the change in $\sum_{u} R(u, v)$ and show that the total change on iteration $t$ is exactly $b_{t+1}(v)-b_{t}(v)$, which is sufficient for our claim. By lines 17 and 18, for each $x$ satisfying $b_{t}(x) \neq 0$ and each $j \in[s]$, the value $\sum_{u} R\left(u, h_{j}(x)\right)$ increases by $b_{t}(x) / s$ and the value $\sum_{u} R(u, x)$ decreases by $b_{t}(x) / s$. For each $x$ with $b_{t}(x) \neq 0$, the $s$ decreases add up to a total of $b_{t}(x)$. As for $b_{t+1}(v)-b_{t}(v)$, a demand of $b_{t}(v)$ is not transferred over to $b_{t+1}(v)$ if $b_{t}(v) \neq 0$, and $b_{t+1}(v)$ increases by $b_{t}(x) / s$ for each $x, j$ with $h_{j}(x)=v$. Altogether, the differences in $\sum_{u} R(u, v)$ and $b_{t+1}(v)-b_{t}(v)$ match.

Invariant 4.18. $R_{t+1}^{*}$ satisfies demand vector $b_{t+1}$.

Proof. Suppose by induction on $t$ that $R_{t}^{*}$ satisfies demand vector $b_{t}$; the base case $t=0$ is trivial. For each $v$ satisfying $b_{t}(v) \neq 0$ and each $j \in[s]$, the value $\sum_{u} R_{t+1}^{*}\left(u, h_{j}(v)\right)$ increases by $\sum_{x, v} R_{t}^{*}(x, v) / s$ (line 21 ), which by induction is exactly $b_{t}(v) / s$. This matches the increase of $b_{t+1}\left(h_{j}(v)\right)$ by $b_{t}(v) / s$ on line 19 .

Invariant 4.19. For each pair $(x, y)$ with $R_{t+1}^{*}(x, y) \neq 0, x-y$ has all coordinates an (integral) multiple of $w^{t}$.

Proof. The only changes to $R_{t+1}^{*}$ are the $R_{t+1}^{*}\left(h_{j}(x), h_{j}(y)\right)$ changes on line 21. By definition of $h_{j}$, we have that $h_{j}(u)-h_{j}(v)$ is a multiple of $W=w^{t}$ for all $u, v$.

Lemma 4.11. The total cost of routing on each iteration $t$ (lines 17 and 18) is at most $k w \cdot \operatorname{cost}\left(R_{t}^{*}\right)$.

Proof. For each trial $j \in[s]$, by construction of $h_{j}(x)$ (line 13), we have $\left\|\left(h_{j}(x)\right)_{i}-x_{i}\right\|_{1} \leq k W$, which incurs a cost of at most $\left|b_{t}(x) / s\right| \cdot k W$ in the routing $R$ (lines 17 and 18). Over all $s$ iterations, each $x \in \mathbb{R}^{k}$ with $b_{t}(x) \neq 0$ is responsible for at most $\left|b_{t}(x)\right| \cdot k W$ cost.

We now bound $\sum_{x}\left|b_{t}(x)\right| \cdot k W$ in terms of $\operatorname{cost}\left(R_{t}^{*}\right)$. By Invariant 4.18 , for each $x$ with $b_{t}(x) \neq 0$,

$$
\sum_{y}\left|R_{t}^{*}(x, y)\right| \geq\left|\sum_{y} R_{t}^{*}(x, y)\right|=\left|b_{t}(x)\right|
$$

(Here, the summation is over the finitely many $y$ that produce a nonzero summand.) Summing over all such $x$, we get

$$
\sum_{x: b_{t}(x) \neq 0}\left|b_{t}(x)\right| \leq \sum_{x: b_{t}(x) \neq 0} \sum_{y}\left|R_{t}^{*}(x, y)\right| \leq \sum_{x, y}\left|R_{t}^{*}(x, y)\right| .
$$

By Invariant 4.19 , we have $\|x-y\|_{1} \geq w^{t-1}$ for each $(x, y)$ with $R_{t}^{*}(x, y) \neq 0$. Therefore,

$$
\operatorname{cost}\left(R_{t}^{*}\right)=\sum_{x, y}\left|R_{t}^{*}(x, y)\right| \cdot\|x-y\|_{1} \geq \sum_{x, y}\left|R_{t}^{*}(x, y)\right| \cdot w^{t-1} .
$$

Thus, the cost is at most

$$
\begin{aligned}
& \sum_{x}\left|b_{t}(x)\right| \cdot k W \stackrel{(2)}{\leq} \sum_{x, y}\left|R_{t}^{*}(x, y)\right| \cdot k W \\
= & k w \cdot \sum_{x, y}\left|R_{t}^{*}(x, y)\right| \cdot w^{t-1} \stackrel{(3)}{\leq} k w \cdot \operatorname{cost}\left(R_{t}^{*}\right) .
\end{aligned}
$$


Claim 4.20. For each $t \in[T+1], R_{t}^{*}$ has support size $n^{O(1)}$.

Proof. For each $t \in[0, T]$, by lines 20 and 21, every $(x, y)$ with $R_{t}^{*}(x, y)$ is responsible for creating at most $s \leq O\left(\log ^{5} n\right)$ nonzero values in $R_{t+1}^{*}$. Also, $R_{0}^{*}$ is supported in $V$, so it has support size $n^{O(1)}$. Therefore, $R_{t}^{*}$ has support size at most

$$
n^{O(1)} \cdot s^{T+1}=n^{O(1)} \cdot\left(O\left(\log ^{5} n\right)\right)^{O(\log n / \log \log n)}=n^{O(1)} .
$$

Lemma 4.21. Fix two points $(x, y)$ with $R_{t+1}^{*}(x, y) \neq 0$, and fix $a$ coordinate $i \in[k]$. With probability $1-n^{-\omega(1)}$, we have

$$
\frac{1}{s} \sum_{j=1}^{s}\left|\left(h_{j}(x)\right)_{i}-\left(h_{j}(y)\right)_{i}\right| \leq\left(1+\frac{1}{\log n}\right)\left|x_{i}-y_{i}\right| .
$$

Proof. Define $\delta_{i}:=x_{i}-y_{i}$. First, if $\delta_{i}=0 \Longleftrightarrow x_{i}=y_{i}$, then $\left(h_{j}(x)\right)_{i}=\left(h_{j}(y)\right)_{i}$ with probability 1 , so both sides of (5) are zero.

Assume now that $\delta_{i}>0$. Throughout the proof, we recommend the reader assume $W=1$ so that $\lfloor x\rfloor_{W}$ is simply $\lfloor x\rfloor$, etc., since the proof is unchanged upon scaling $W$. Define $\{x\}_{W}:=x-\lfloor x\rfloor_{W}$, the "remainder" of $x$ when divided by $W$.

Observe that for each of the $s$ independent trials, $\left(h_{j}(x)\right)_{i}-$ $\left(h_{j}(y)\right)_{i}=\left\lfloor\delta_{i}\right\rfloor_{W}$ with probability $1-\left\{\delta_{i}\right\}_{W} / W$ and $\left(h_{j}(x)\right)_{i}-$ $\left(h_{j}(y)\right)_{i}=\left\lceil\delta_{i}\right\rceil_{W}$ with probability $\left\{\delta_{i}\right\}_{W} / W$. In particular, $\mathbb{E}\left[\left(h_{j}(x)\right)_{i}-\right.$ $\left.\left(h_{j}(y)\right)_{i}\right]=\delta_{i}$.

For $j \in[s]$, define random variable $X_{j}$ as the value of $\left(\left(h_{j}(x)\right)_{i}-\right.$ $\left.\left(h_{j}(y)\right)_{i}-\left\lfloor\delta_{i}\right\rfloor_{W}\right) / W$ on the $j$ 'th independent trial, so that $X_{j} \in$ $\{0,1\}$ and $\mathbb{E}\left[X_{j}\right]=\left\{\delta_{i}\right\}_{W} / W$ for all $j$. We can express the LHS of (5) as

$$
\begin{aligned}
\frac{1}{s} \sum_{j=1}^{s}\left|\left(h_{j}(x)\right)_{i}-\left(h_{j}(y)\right)_{i}\right| & =\frac{1}{s} \sum_{j=1}^{s}\left(\left(h_{j}(x)\right)_{i}-\left(h_{j}(y)\right)_{i}\right) \\
& =\frac{1}{s} \sum_{j=1}^{s}\left(W \cdot X_{j}+\left\lfloor\delta_{i}\right\rfloor_{W}\right) \\
& =\frac{W}{s} \sum_{j=1}^{s} X_{j}+\left\lfloor\delta_{i}\right\rfloor_{W} \\
& =\frac{W}{s} \sum_{j=1}^{s} X_{j}+\left(\delta_{i}-\left\{\delta_{i}\right\}_{W}\right) .
\end{aligned}
$$

Define $\mu:=\sum_{j} \mathbb{E}\left[X_{j}\right]=(s / W)\left\{\delta_{i}\right\}_{W}$. By Invariant 4.19 applied to iteration $t-1$, we know that $\delta_{i}$ is a multiple of $w^{t-1}=W / w$, so $\left\{\delta_{i}\right\} \geq W / w$, which means $\mu \geq s / w$. Applying Chernoff bounds on the variables $X_{1}, \ldots, X_{s} \in[0,1]$ with $\epsilon:=1 / \log n$, we obtain

$$
\begin{gathered}
\operatorname{Pr}\left[\sum_{j=1}^{s} X_{j} \geq(1+\epsilon) \mu\right] \leq \exp \left(-\epsilon^{2} \mu / 3\right) \leq \exp \left(-\frac{s}{3 w \log ^{2} n}\right) \\
=\exp (-\omega(\log n))=n^{-\omega(1)} .
\end{gathered}
$$

This means that with probability $1-n^{\omega(1)}$,

$$
\begin{aligned}
\frac{1}{s} \sum_{j=1}^{s}\left|\left(h_{j}(x)\right)_{i}-\left(h_{j}(y)\right)_{i}\right| & \stackrel{(6)}{=} \frac{W}{s} \sum_{j=1}^{s} X_{j}+\left(\delta_{i}-\left\{\delta_{i}\right\}_{W}\right) \\
& \leq \frac{W}{s}(1+\epsilon) \mu+\left(\delta_{i}-\left\{\delta_{i}\right\}_{W}\right) \\
& =(1+\epsilon)\left\{\delta_{i}\right\}_{W}+\delta_{i}-\left\{\delta_{i}\right\}_{W}
\end{aligned}
$$

$$
\begin{aligned}
& =\delta_{i}+\epsilon\left\{\delta_{i}\right\}_{W} \\
& \leq(1+\epsilon) \delta_{i} \\
& =\left(1+\frac{1}{\log n}\right)\left|x_{i}-y_{i}\right|,
\end{aligned}
$$

completing (5).

Finally, for the case $\delta_{i}<0$, we can simply swap $x$ and $y$ and use the $\delta_{i}>0$ case.

Lemma 4.12. With probability $1-n^{-\omega(1)}, \operatorname{cost}\left(R_{t+1}^{*}\right) \leq(1+$ $\left.\frac{1}{\log n}\right) \operatorname{cost}\left(R_{t}^{*}\right)$ for each iteration $t$.

Proof. By lines 20 and 21, every $(x, y)$ with $R_{t}^{*}(x, y) \neq 0$ is responsible for a total cost of

$$
\begin{gathered}
\sum_{j=1}^{s} \frac{\left|R_{t}^{*}(x, y)\right|}{s} \cdot\left\|h_{j}(x)-h_{j}(y)\right\|_{1} \\
=\frac{\left|R_{t}^{*}(x, y)\right|}{s} \sum_{j=1}^{s} \sum_{i=1}^{k}\left|\left(h_{j}(x)\right)_{i}-\left(h_{j}(y)\right)_{i}\right| .
\end{gathered}
$$

We now take a union bound over all such $(x, y)$ (at most $n^{O(1)}$ many by Claim 4.20). By Lemma 4.21 , we have that with probability $1-n^{-\omega(1)}$, the total cost is at most

$$
\begin{aligned}
& \frac{\left|R_{t}^{*}(x, y)\right|}{s} \sum_{j=1}^{s} \sum_{i=1}^{k}\left(1+\frac{1}{\log n}\right)\left|x_{i}-y_{i}\right| \\
& =\left(1+\frac{1}{\log n}\right)\left|R_{t}^{*}(x, y)\right| \cdot\|x-y\|_{1} .
\end{aligned}
$$

Summing over all such $(x, y)$, we obtain

$$
\operatorname{cost}\left(R_{t+1}^{*}\right) \leq\left(1+\frac{1}{\log n}\right) \operatorname{cost}\left(R_{t}^{*}\right),
$$

as desired.

\subsubsection{Proof of Sparsity.}

Definition 4.22 (History graph; originate). Define the history graph $H$ to be the following digraph on vertex set $V(H):=\left(V_{0} \times\right.$ $\{0\}) \cup\left(V_{1} \times\{1\}\right) \cup \cdots \cup\left(V_{T+1} \times\{T+1\}\right)$. For every $t \in\{0,1, \ldots, T\}$ and every $x, y$ such that line 17 is executed at least once on $R(x, y)$, add a directed edge $((x, t),(y, t+1))$ in $H$. (By Invariant 4.23 below, every such $x, y$ must satisfy $x \in V_{t}$ and $y \in V_{t+1}$.) A vertex $(x, t) \in V(H)$ originates from vertex $v \in V=V_{0}$ if there is a directed path in $H$ from $(v, 0)$ to $(x, t)$.

Invariant 4.23. For each $x$ with $b_{t+1}(x) \neq 0$, we have $x \in V_{t+1}$.

Proof. Every $x \in V$ with value $b_{t+1}(x)$ modified in line 19 is added into $V_{t+1}$ in line 17.

Invariant 4.24. For each point $v \in V$ and point $x \in V_{t}$ where $(x, t)$ originates from $v$,

$$
\forall i \in[k]: 0 \leq v_{i}-x_{i} \leq \sum_{j=1}^{t} w^{j}
$$


Proof. We prove the statement by induction on $t$; the base case $t=0$ is trivial. For iteration $t$, for each $v, x$ where $(x, t)$ originates from $v$, we have $x_{i}-W<\left(h_{j}(x)\right)_{i} \leq x_{i}$ for all $i \in[k], j \in[s]$ by definition of $h_{j}$. By induction, $0 \leq v_{i}-x_{i} \leq \sum_{j=1}^{t-1} w^{j}$ for all $i \in[k]$. Therefore, the points $h_{j}(x) \in V_{t+1}$, which also originate from $v$, satisfy

$$
v_{i}-\left(h_{j}(x)\right)_{i} \geq v_{i}-x_{i} \geq 0
$$

and

$$
v_{i}-\left(h_{j}(x)\right)_{i} \leq v_{i}-x_{i}+W \leq \sum_{j=1}^{t-1} w^{j}+W=\sum_{j=1}^{t} w^{j}
$$

for all $i \in[k]$, completing the induction.

Lemma 4.25. For each point $v \in V$ and iteration $t \in[T+1]$, the expected number of vertices $(x, t) \in V(H)$ that originate from $v$ is $O(s)$.

Proof. Fix an iteration $t \in[T+1]$. Let $r:=\sum_{j=1}^{t-1} w^{j} \leq 2 w^{t-1}$; by Invariant 4.24, all points $x \in V_{t}$ such that $(x, t)$ originates from $v$ are within the box $B:=\left[v_{1}-r, v_{1}\right] \times\left[v_{2}-r, v_{2}\right] \times \cdots \times\left[v_{k}-r, v_{k}\right]$.

For each trial $j \in[s]$, consider the set $S:=\left\{h_{j}(x): x \in B\right\}$; note that every $y$ in lines 17 and 18 for this trial satisfies $y \in S$. We claim that this set has expected size $O(1)$. To see why, observe that for each $i \in[k]$, the value $\left(h_{j}(x)\right)_{i}$ over all $x \in B$ takes two distinct values with probability $r / W$ and one value with probability $1-r / W$, and these events are independent over all $i$. Moreover, if $k^{\prime} \leq k$ of them take two distinct values, then $|S| \leq 2^{k^{\prime}}$, and this happens with probability $\left(\begin{array}{c}k \\ k^{\prime}\end{array}\right)\left(\frac{r}{W}\right)^{k^{\prime}}\left(1-\frac{r}{W}\right)^{k-k^{\prime}}$. Overall, the expected size of $|S|$ is at most

$$
\begin{aligned}
\sum_{k^{\prime}=0}^{k}\left(\begin{array}{c}
k \\
k^{\prime}
\end{array}\right)\left(\frac{r}{W}\right)^{k^{\prime}}\left(1-\frac{r}{W}\right)^{k-k^{\prime}} \cdot 2^{k^{\prime}} & \\
= & \left(\frac{r}{W} \cdot 2+\left(1-\frac{r}{W}\right)\right)^{k}=\left(1+\frac{r}{W}\right)^{k} \\
& \leq\left(1+\frac{2 w^{t-1}}{w^{t}}\right)^{k}=\left(1+\frac{2}{\lceil\log n\rceil}\right)^{O(\log n)}=O(1)
\end{aligned}
$$

Over all $s$ independent trials, the sets $S$ together capture all points $y$ such that $(y, t)$ originates from $v$. The expected number of such points $(y, t)$ is therefore at most $O(s)$.

\subsubsection{Proof of Last Routing (lines 24 and 25).}

Lemma 4.13. The total cost of routing on lines 24 and 25 is at most $O(k w) \cdot \operatorname{cost}\left(R_{T+1}^{*}\right)$.

Proof. We can follow the proof of Lemma 4.11 to obtain (4), where $W:=w^{T+1}$ in this case. By Invariant 4.24, for each point $v \in V$ and point $x \in V_{T+1}$ where $(x, t)$ originates from $v$, we have $\|v-x\|_{1} \leq k w \sum_{j=1}^{T} w^{j}=O\left(k w^{T+1}\right)$. By Assumption 4.6, the vertices $v \in V$ are at most $n^{c} \leq w^{T}$ apart from each other in $\ell_{1}$ distance. This means that the points $x \in V_{T+1}$ are at most $O\left(k w^{T+1}\right)$ apart in $\ell_{1}$ distance. Therefore, the routing on lines 24 and 25 has cost $C \leq \sum_{x}\left|b_{T+1}(x)\right| \cdot O\left(k w^{T+1}\right)$. Combining this with (4) gives

$$
k w \operatorname{cost}\left(R^{*}\right) \geq \sum_{x}\left|b_{T+1}(x)\right| \cdot k w^{T+1} \geq \Omega(C),
$$

which means $C \leq O(k w) \cdot \operatorname{cost}\left(R_{T+1}^{*}\right)$, as desired.
4.5.5 Computing the Matrix R. First, we can modify Algorithm 1 to construct the graph $H$ without changing the running time, since every edge added to $H$ can be charged to one execution of line 17 .

Now for any vector $b \in \mathbb{R}^{V}$ not necessarily satisfying $1 \cdot b=0$, let $R_{b}$ be the value of $R$ once Algorithm 1 is run on $b$. First, we will henceforth assume the following for simplicity:

Assumption 4.26. For each $b$, every $(x, y)$ is updated at most once in $R_{b}(x, y)$ throughout Algorithm 1.

Intuitively, Assumption 4.26 is true with probability 1 because two different randomly shifted grids in Algorithm 1 align perfectly with probability 0 . More specifically, the probability that $h_{j}(x)=$ $h_{j^{\prime}}\left(x^{\prime}\right)$ for two distinct $x, j$ and $x^{\prime}, j^{\prime}$ (possibly not even at the same iteration) is 0 .

Lemma 4.27. Assuming Assumption 4.26, we have that w.h.p., for each $b$ and iteration $t$,

$$
\begin{gathered}
\frac{1}{4} k w^{t} \sum_{x: b_{t}(x) \neq 0}\left|b_{t}(x)\right| \leq \sum_{x, y: b_{t}(x) \neq 0}\left|R_{b}(x, y)\right| \cdot\|x-y\|_{1} \\
\leq \frac{3}{4} k w^{t} \sum_{x: b_{t}(x) \neq 0}\left|b_{t}(x)\right|
\end{gathered}
$$

Proof. Fix some $x \in V_{t}$, and fix a coordinate $i \in[k]$. For each trial $j \in[s]$, the difference $x_{i}-\left(h_{j}(x)\right)_{i}$ is a uniformly random number in $[0, W)$ (where $W:=w^{t}$ as before). Define random variable $X_{j}:=\left(x_{i}-\left(h_{j}(x)\right)_{i}\right) / W \in[0,1]$, and $\mu:=\sum_{j} \mathbb{E}\left[X_{j}\right]=s / 2$. Applying Chernoff bounds on the variables $X_{j}$ with $\epsilon:=1 / 4$, we obtain

$$
\operatorname{Pr}\left[\left|\sum_{j=1}^{s} X_{j}-\mu\right| \geq \epsilon \mu\right] \leq \exp \left(-\epsilon^{2} \mu / 3\right) \leq \exp (-\Omega(s))=n^{-\omega(1)}
$$

Therefore, with probability $1-n^{-\omega(1)}$,

$$
\sum_{j=1}^{s}\left(x_{i}-\left(h_{j}(x)\right)_{i}\right)=\sum_{j=1}^{s} W X_{i} \in\left[\frac{s}{4} W, \frac{3 s}{4} W\right]
$$

Summing over all $i \in[k]$, we obtain

$$
\sum_{j=1}^{s}\left\|x-h_{j}(x)\right\|_{1}=\sum_{j=1}^{s} \sum_{i=1}^{k}\left(x_{i}-\left(h_{j}(x)\right)_{i}\right) \in\left[\frac{1}{4} k s W, \frac{3}{4} k s W\right] .
$$

At this point, let us assume that every statement holds in the proof so far, which is true w.h.p. Fix a demand vector $b$; by Assumption 4.26, each term in the sum $\sum_{x, y: b_{t}(x) \neq 0}\left|R_{b}(x, y)\right| \cdot\|x-y\|_{1}$ appears exactly once in line 17 , so it must appear on iteration $t$. In particular, the terms can be exactly partitioned by $x$. Every $x$ with $b_{t}(x) \neq 0$ contributes $\sum_{j=1}^{s}\left|b_{t}(x) / s\right| \cdot\left\|x-h_{j}(x)\right\|_{1}$ to the sum (line 17), which is within $\left[\frac{1}{4} k W\left|b_{t}(x)\right|, \frac{3}{4} k W\left|b_{t}(x)\right|\right]$. Summing over all $x$ proves (7).

Therefore, by Lemma 4.27, to estimate the final routing cost $\sum_{x, y}\left|R_{b}(x, y)\right| \cdot\|x-y\|_{1}$ by an $O(1)$ factor, it suffices to compute the value

$$
\sum_{t} k w^{t} \sum_{x: b_{t}(x) \neq 0}\left|b_{t}(x)\right|
$$


Remark 4.28. The purpose of reducing to summing over the values $\left|b_{t}(x)\right|$ is to save a factor $s$ in the running time; if we did not care about extra $\operatorname{polylog}(n)$ factors, we could do without it.

Assuming Assumption 4.26, our goal is to construct a sparse matrix $R$ so that $\|R b\|_{1}$ equals (8). To do so, our goal is to have each coordinate in $R b$ represent $k w^{t} b_{t}(x)$ for some $t, x$ with $b_{t}(x) \neq 0$. This has the benefit of generalizing to general demands $b$ by the following linearity property:

Claim 4.29. Every value $b_{t}(x)$ for $t \in\{0,1,2, \ldots, T+1\}, x \in \mathbb{R}^{k}$ is a linear function in the entries of $b \in \mathbb{R}^{V}$.

Proof. We show this by induction on $t$; the base case $t=0$ is trivial. For each $t>0$, the initialization $b_{t+1}$ as the zero function is linear in $b$, and by line 19 , each update of some $b_{t+1}(y)$ adds a scalar multiple of some $b_{t}(x)$ to $b_{t+1}(y)$. Since $b_{t+1}(y)$ was linear in $b$ before the operation, and since $b_{t}(x)$ is linear in $b$ by induction, $b_{t+1}(y)$ remains linear in $b$.

To exploit linearity, we consider the set of "basis" functions $R_{b}$ where $b=\chi_{v}$ for some $v \in V$. (Again, note that $\chi_{v}$ is not a demand vector, but we do not require that property here.)

Lemma 4.15. For each $\chi_{v}$, if we run Algorithm 1 on demands $\chi_{v}$, every function $b_{t}$ has $O(s)$ nonzero values in expectation. Moreover, each function $b_{t}$ can be computed in $O\left(s^{2}\right)$ expected time.

Proof. We first show by induction on $t$ that if $b_{t}(x) \neq 0$ for $x \in \mathbb{R}^{k}$, then $(x, t)$ originates from $v$; the base case $t=0$ is trivial. For each $t>0$, the only way some $b_{t+1}(y)$ is updated (line 19) is if there exist $x \in \mathbb{R}^{k}$ with $b_{t}(x) \neq 0$ and $y=h_{j}(x)$ for some $j \in[s]$. By induction, $x$ originates from $v$, and by definition of the history graph $H$, there is a directed edge $((x, t),(y, t+1))$ in $H$ added when line 17 is executed for this pair $x, y$. Therefore, there is a path from $(v, 0)$ to $(y, 1)$ in $H$, and $y$ also originates from $v$.

Therefore, for each $t$, the number of points $x \in \mathbb{R}^{k}$ satisfying $b_{t}(x)$ is at most the number of vertices $(x, t) \in V(H)$ originating from $v$, which by Lemma 4.25 is $O(s)$ in expectation.

Finally, the functions $b_{t}$ can be computed by simply running Algorithm 1. $O(s)$ time is spent for each $(x, t)$ with $b_{t}(x) \neq 0$ (assuming the entries of $R_{\chi_{v}}$ are stored in a hash table), giving $O\left(s^{2}\right)$ expected time for each iteration $t$.

Lemma 4.16. We can compute a matrix $R$ such that $\|R b\|_{1}$ approximates the cost of routing in Algorithm 1 to factor $O(1)$, and $R$ has $O(s T n)=O\left(n \log ^{5} n(\log \log n)^{O(1)}\right)$ nonzero entries. The algorithm succeeds w.h.p., and runs in time $O\left(s^{2} T n \log n\right)=O\left(n \log ^{10} n(\log \log n)^{O(1)}\right)$.

Proof. We run Algorithm 1 for each demand $\chi_{v}$ over the same randomness (in particular, the same choices of $h_{j}$ ); define $b_{t}^{\chi_{v}}$ to be the function $b_{t}$ on input $\chi_{v}$. Let $b_{t}$ be the functions on input $b$. By linearity (Claim 4.29), we have that for each $t, x$,

$$
b_{t}(x)=\sum_{v \in V} b(v) \cdot b_{t}^{\chi_{v}}(x)
$$

By Lemma 4.15, the functions $b_{t}^{\chi_{v}}$ for all $t, \chi_{v}$ can be computed in $O\left(s^{2} T n\right)$ total time in expectation.
We now construct matrix $R$ as follows: for each $t, x$ with $b_{t}^{\chi_{v}}(x) \neq$ 0 for at least one $\chi_{v}$, we add a row to $R$ with value $k w^{t} b_{t}^{\chi_{v}}$ at each entry $v \in V$. The dot product of this row with $b$, which becomes a coordinate entry in $R b$, is exactly

$$
\sum_{v \in V} k w^{t} b_{t}^{\chi v}(x) \cdot b(v) \stackrel{(9)}{=} k w^{t} b_{t}(x) .
$$

Hence, $\|R b\|_{1}$ is exactly (8), which approximates the routing cost to factor $O(1)$ by Lemma 4.27, assuming Assumption 4.26 (which holds with probability 1 ). Finally, by Lemma $4.15, R$ has $O(s T n)$ entries in expectation.

Lastly, we address the issue that the algorithm only runs quickly in expectation, not w.h.p. Our solution is standard: run the algorithm $O(\log n)$ times, terminating it early each time if the running time exceeds twice the expectation. Over $O(\log n)$ tries, one will finish successfully w.h.p., so the final running time has an extra factor of $O(\log n)$, hence $O\left(s^{2} T n \log n\right)$.

\subsection{Parallel Transshipment}

By inspection, the entire Algorithm 1 is parallelizable in $\widetilde{O}(m)$ work and polylog $(n)$ time. The only obstacle to the entire $\ell_{1}$-oblivious routing algorithm is the initial $\ell_{1}$-embedding step, and the only hurdle to the final proof of Theorem 1.4 is the final low-stretch spanning step. The latter we can handle with Remark 4.3, since minimum spanning tree can be computed in parallel with Boruvka's algorithm. We state the following corollary below to be used in our parallel algorithms.

Corollary 3.3. Given an undirected graph with nonnegative weights and polynomial aspect ratio, and given an $\ell_{1}$-embedding of the graph with polylog $(n)$ distortion in $O(\log n)$ dimensions, there is a parallel algorithm to compute $a(1+\epsilon)$-approximate minimum transshipment instance in $\widetilde{O}\left(m \epsilon^{-2}\right)$ work and polylog $(n) \epsilon^{-2}$ time.

\section{ACKNOWLEDGMENTS}

The author thanks Yasamin Nazari, Thatchaphol Saranurak, and Goran Zuzic for helpful discussions, and Richard Peng for useful comments on multiple drafts of the paper. The author thanks Alexandr Andoni, Cliff Stein, and Peilin Zhong for pointing out a nontrivial omission of the first version of this paper, namely an issue in rounding the transshipment flow to an "expected" SSSP, which was later resolved by the introduction of a new section in the full version of this paper. This section was written with only the knowledge of their work's existence (namely, that another group had solved parallel $s-t$ path), and without knowing any of their techniques or proofs. The remaining sections remain fully independent of their work.

\section{REFERENCES}

[AN12] Ittai Abraham and Ofer Neiman. Using petal-decompositions to build a low stretch spanning tree. In Proceedings of the forty-fourth annual ACM symposium on Theory of computing, pages 395-406. ACM, 2012.

[ASZ19] Alexandr Andoni, Clifford Stein, and Peilin Zhong. Parallel approximate undirected shortest paths via low hop emulators. arXiv preprint arXiv:1911.01956, 2019.

[BK13] Ruben Becker and Andreas Karrenbauer. A combinatorial o(m 3/2)-time algorithm for the min-cost flow problem. arXiv preprint arXiv:1312.3905, 2013. 
[BKKL16] Ruben Becker, Andreas Karrenbauer, Sebastian Krinninger, and Christoph Lenzen. Near-optimal approximate shortest paths and transshipment in distributed and streaming models. arXiv preprint arXiv:1607.05127, 2016.

[CKM ${ }^{+}$11] Paul Christiano, Jonathan A Kelner, Aleksander Madry, Daniel A Spielman, and Shang-Hua Teng. Electrical flows, laplacian systems, and faster approximation of maximum flow in undirected graphs. In Proceedings of the forty-third annual ACM symposium on Theory of computing, pages 273-282. ACM, 2011.

[CMSV17] Michael B Cohen, Aleksander Madry, Piotr Sankowski, and Adrian Vladu. Negative-weight shortest paths and unit capacity minimum cost flow in õ (m 10/7 log w) time*. In Proceedings of the Twenty-Eighth Annual ACMSIAM Symposium on Discrete Algorithms, pages 752-771. SIAM, 2017.

[Coh00] Edith Cohen. Polylog-time and near-linear work approximation scheme for undirected shortest paths. Fournal of the ACM ( $\mathcal{A} A C M), 47(1): 132-166$, 2000

[DS08] Samuel I Daitch and Daniel A Spielman. Faster approximate lossy generalized flow via interior point algorithms. In Proceedings of the fortieth annual ACM symposium on Theory of computing, pages 451-460. ACM, 2008

[EN18] Michael Elkin and Ofer Neiman. Efficient algorithms for constructing very sparse spanners and emulators. ACM Transactions on Algorithms (TALG) 15(1):4, 2018.

[Fin18] Jeremy T Fineman. Nearly work-efficient parallel algorithm for digraph reachability. In Proceedings of the 50th Annual ACM SIGACT Symposium on Theory of Computing, pages 457-470. ACM, 2018.

[FN18] Sebastian Forster and Danupon Nanongkai. A faster distributed singlesource shortest paths algorithm. In 2018 IEEE 59th Annual Symposium on Foundations of Computer Science (FOCS), pages 686-697. IEEE, 2018.

[HP11] Sariel Har-Peled. Geometric approximation algorithms. Number 173. American Mathematical Soc., 2011.

[JL84] William B Johnson and Joram Lindenstrauss. Extensions of lipschitz mappings into a hilbert space. Contemporary mathematics, 26(189-206):1, 1984

[KLOS14] Jonathan A Kelner, Yin Tat Lee, Lorenzo Orecchia, and Aaron Sidford. An almost-linear-time algorithm for approximate max flow in undirected graphs, and its multicommodity generalizations. In Proceedings of the twenty-fifth annual ACM-SIAM symposium on Discrete algorithms, pages 217-226. SIAM, 2014.

[KMP14a] Ioannis Koutis, Gary L Miller, and Richard Peng. Approaching optimality for solving sdd linear systems. SIAM fournal on Computing, 43(1):337-354, 2014

[KMP14b] Ioannis Koutis, Gary L Miller, and Richard Peng. Approaching optimality for solving sdd linear systems. SIAM fournal on Computing, 43(1):337-354, 2014

[KNP19] Andrey Boris Khesin, Aleksandar Nikolov, and Dmitry Paramonov. Preconditioning for the geometric transportation problem. arXiv preprint arXiv:1902.08384, 2019

[KS92] Philip N Klein and Sairam Subramanian. A parallel randomized approximation scheme for shortest paths. In STOC, volume 92, pages $750-758$,
1992.

[KS97] Philip N Klein and Sairam Subramanian. A randomized parallel algorithm for single-source shortest paths. Journal of Algorithms, 25(2):205-220, 1997.

[LLR95] Nathan Linial, Eran London, and Yuri Rabinovich. The geometry of graphs and some of its algorithmic applications. Combinatorica, 15(2):215-245, 1995.

[LS14] Yin Tat Lee and Aaron Sidford. Path finding methods for linear programming: Solving linear programs in o (vrank) iterations and faster algorithms for maximum flow. In 2014 IEEE 55th Annual Symposium on Foundations of Computer Science, pages 424-433. IEEE, 2014.

[Mad10] Aleksander Madry. Fast approximation algorithms for cut-based problems in undirected graphs. In FOCS, pages 245-254. IEEE Computer Society, 2010.

[Mad13] Aleksander Madry. Navigating central path with electrical flows: From flows to matchings, and back. In 2013 IEEE 54th Annual Symposium on Foundations of Computer Science, pages 253-262. IEEE, 2013.

[MPVX13] Gary L Miller, Richard Peng, Adrian Vladu, and Shen Chen Xu. Improved parallel algorithms for spanners and hopsets. arXiv preprint arXiv:1309.3545, 2013.

[Nes05] Yu Nesterov. Smooth minimization of non-smooth functions. Mathematical programming, 103(1):127-152, 2005.

[Pen16] Richard Peng. Approximate undirected maximum flows in o (m polylog (n)) time. In Proceedings of the twenty-seventh annual ACM-SIAM symposium on Discrete algorithms, pages 1862-1867. SIAM, 2016.

[PS14] Richard Peng and Daniel A Spielman. An efficient parallel solver for sdd linear systems. In Proceedings of the forty-sixth annual ACM symposium on Theory of computing, pages 333-342. ACM, 2014.

[Räc08] Harald Räcke. Optimal hierarchical decompositions for congestion minimization in networks. In Proceedings of the fortieth annual ACM symposium on Theory of computing, pages 255-264. ACM, 2008.

[Ren88] James Renegar. A polynomial-time algorithm, based on newton's method, for linear programming. Mathematical Programming, 40(1-3):59-93, 1988.

[RST14] Harald Räcke, Chintan Shah, and Hanjo Täubig. Computing cut-based hierarchical decompositions in almost linear time. In Proceedings of the twenty-fifth annual ACM-SIAM symposium on Discrete algorithms, pages 227-238. Society for Industrial and Applied Mathematics, 2014.

[She13] Jonah Sherman. Nearly maximum flows in nearly linear time. In 2013 IEEE 54th Annual Symposium on Foundations of Computer Science, pages 263-269. IEEE, 2013.

[She17a] Jonah Sherman. Area-convexity, lấlđ regularization, and undirected multicommodity flow. In Proceedings of the 49th Annual ACM SIGACT Symposium on Theory of Computing, pages 452-460. ACM, 2017.

[She17b] Jonah Sherman. Generalized preconditioning and undirected minimumcost flow. In Proceedings of the Twenty-Eighth Annual ACM-SIAM Symposium on Discrete Algorithms, pages 772-780. SIAM, 2017.

[ST04] Daniel A Spielman and Shang-Hua Teng. Nearly-linear time algorithms for graph partitioning, graph sparsification, and solving linear systems. In Proceedings of the STOC, volume 4, 2004.

[Ye97] Yinyu Ye. Interior point algorithms: theory and analysis. Springer, 1997. 